\title{
FLUCTUATIONS OF BROWNIAN MOTION WITH DRIFT
}

\author{
Joseph G. Conlon* and Peder Olsen**
}

\begin{abstract}
Consider 3 dimensional Brownian motion started on the unit sphere $\{|x|=1\}$ with initial density $\rho$. Let $\rho_{t}$ be the first hitting density on the sphere $\{|x|=t+1\}, t>0$. Then the linear operators $T_{t}$ defined by $T_{t} \rho=\rho_{t}$ form a semigroup with an infinitesimal generator which is approximately the square root of the Laplacian. This paper studies the analogous situation for Brownian motion with a drift $\mathbf{b}$, where $\mathbf{b}$ is small in a suitable scale invariant norm.
\end{abstract}

\section{Chapter 1. Introduction}

In two previous papers $[\mathbf{C R}],[\mathbf{C O}]$ we studied the Dirichlet problem for an elliptic equation on a domain in $\mathbb{R}^{3}$. Let $B_{R}$ be the ball of radius $R$ in $\mathbb{R}^{3}$ centered at the origin, $0<R<\infty$. Consider the problem

$$
\begin{aligned}
(-\Delta-\mathbf{b}(x) \cdot \nabla) u(x) & =f(x), & & x \in B_{R}, \\
u(x) & =0, & & x \in \partial B_{R} .
\end{aligned}
$$

A function $g: \mathbb{R}^{3} \rightarrow \mathbb{C}$ is said to be in the Morrey space $M_{p}^{q}$, $1 \leq p \leq q<\infty$, if $|g|^{p}$ is locally integrable and there is a constant $C$ such that

$$
\int_{Q}|g|^{p} d x \leq C^{p}|Q|^{1-p / q}
$$

for all cubes $Q \subset \mathbb{R}^{3}$. Here $|Q|$ denotes the volume of $Q$. The norm of $g,\|g\|_{q, p}$ is defined as the minimum $C$ for which (1.2) holds. In $[\mathbf{C R}]$ we proved the following:

* Research partially supported by the U.S. National Science Foundation under grants DMS 9403399 and DMS 9404197.

** Research supported by NFR - Norges Forskingsråd (Norwegian Research Council) grant 100222/40. 
Theorem 1.1. Suppose $1<r<p \leq q, 1<p \leq 3$, and $|\mathbf{b}| \in M_{p}^{3}$, $f \in M_{r}^{q}$, for some $q$ with $q>3 / 2$. Then there exists $\epsilon>0$ depending only on $r, p, q$ such that if $\|\mathbf{b}\|_{3, p}<\epsilon$ then the Dirichlet problem (1.1) is solvable. Further, there is a constant $C$ depending only on $r, p, q$ such that the solution $u$ of (1.1) satisfies the inequality,

$$
\|u\|_{\infty} \leq C R^{2-3 / q}\|f\|_{q, r} .
$$

The condition $\|\mathbf{b}\|_{3, p}$ small for some $p, 1<p \leq 3$, includes the two important cases when $|\mathbf{b}(x)|=\epsilon /|x|$ and $|\mathbf{b}| \in L^{3}\left(\mathbb{R}^{3}\right),\|\mathbf{b}\|_{3}=\epsilon$, $\epsilon \ll 1$. Theorem 1.1 is a perturbative result. Writing the solution of (1.1) as a perturbation series in $\mathbf{b}$ one can show that the series converges if $\|\mathbf{b}\|_{3, p} \ll 1$. Observe also that Theorem 1.1 is a scale invariant theorem. The result for general $R$ can be obtained by a scaling argument from the result for a particular value of $R$. Since all the results in this paper have the same scaling property we shall take $R=1 / 4$ from here on.

It is well known $[\mathbf{S V}]$ that the solution of the Dirichlet problem (1.1) has a representation as an expectation value with respect to Brownian motion with drift $\mathbf{b}$. Let $X_{\mathbf{b}}(t), t \geq 0$, denote the drift process started at time 0 . If $X_{\mathbf{b}}(0) \in B_{1 / 4}$ let $\tau$ be the minimum time $t$ such that $X_{\mathbf{b}}(t) \in \partial B_{1 / 4}$. Then the solution $u$ of (1.1) is given by

$$
u(x)=E_{x}\left[\int_{0}^{\tau} f\left(X_{\mathbf{b}}(t)\right) d t\right], \quad x \in B_{1 / 4}
$$

where $E_{x}$ denotes that the expectation value is taken conditioned on $X_{\mathbf{b}}(0)=x$. Theorem 1.1 tells us therefore something about the behavior of the diffusion process $X_{\mathbf{b}}(t)$ when $\|\mathbf{b}\|_{3, p} \ll 1$. It gives us similar estimates on the expected time the diffusion spends in a subset of $B_{1 / 4}$ before exiting $\partial B_{1 / 4}$ to those one has for standard Brownian motion.

In $[\mathbf{C O}]$ we proved a nonperturbative version of Theorem 1.1, allowing b to be only locally in a Morrey space $M_{p}^{3}$ with small norm. A key ingredient in the proof of this theorem was the fact that the fluctuations of $X_{\mathbf{b}}(t)$ did not increase as $t$ increases, provided $\|\mathbf{b}\|_{3, p} \ll 1$. To be specific, suppose $0<\rho \leq 1 / 2$, and the process $X_{\mathbf{b}}(t)$ starts on the sphere $\partial B_{(1-\rho) / 4}$ with initial density function $f$, and $f_{\rho, \mathbf{b}}$ is the first hitting density on the sphere $\partial B_{1 / 4}$. It is evident, by conservation of probability, that the average value of $f$ is the same as the average value of $f_{\rho, \mathbf{b}}, A v f=A v f_{\rho, \mathbf{b}}$. In [CO] we proved the following: 
Theorem 1.2. Suppose $0<\rho \leq 1 / 2$ and $1<p \leq 3$. Consider $f$ and $f_{\rho, \mathbf{b}}$ to be functions on the unit sphere $S$, with $f \in L^{2}(S)$. Then for $\delta>0$ there exists $\epsilon$, depending only on $p, \rho, \delta$ such that, if $\|\mathbf{b}\|_{3, p}<\epsilon$ and $\|f-A v f\|_{2} \leq \delta|A v f|$, one has $f_{\rho, \mathbf{b}} \in L^{2}(S)$ and $\left\|f_{\rho, \mathbf{b}}-A v f_{\rho, \mathbf{b}}\right\|_{2} \leq$ $\delta\left|A v f_{\rho, \mathbf{b}}\right|$.

It is easy to see that Theorem 1.2 holds uniformly in $\rho$ as $\rho \rightarrow 0$ for the case of Brownian motion, $\mathbf{b} \equiv 0$. Since $\mathbf{b}$ with $\|\mathbf{b}\|_{3, p} \ll 1$ is perturbative to Brownian motion it is natural to expect a similar uniformity when $\|\mathbf{b}\|_{3, p}$ is small. In this paper we prove a uniform version of Theorem 1.2. We cannot however use the $L^{2}$ norm to measure the oscillation of $f$ and $f_{\rho, \mathbf{b}}$. We must use a finer norm which weights high Fourier modes more than low Fourier modes. This subtlety is closely related to the extra complication in the proof of Theorem 1.2 over Theorem 1.1. To prove Theorem 1.1 one shows that a certain integral operator is bounded on Morrey spaces. To prove Theorem 1.2 one needs to know that this same integral operator is bounded on a weighted Morrey space, where the weight of a point $x \in B_{1 / 4}$ decreases as $x$ gets close to $\partial B_{1 / 4}$.

To define our new norm on functions $f$ with domain $S$, let $\Delta_{S}$ be the Laplace operator on the unit sphere. For $k=1,2, \ldots$, let $E_{k}$ be the $L^{2}$ projection operator onto the space spanned by the eigenfunctions of $-\Delta_{S}$ with eigenvalues $\lambda^{2}$ satisfying $2^{k-1}<\lambda \leq 2^{k}$. Let $E_{0}$ be the projection onto the constant function. For $f: S \rightarrow \mathbb{C}$ and $\nu>0$ we define $\|f\|_{2, \nu}$ by

$$
\|f\|_{2, \nu}=\sup _{k \geq 0} 2^{\nu k}\left\|E_{k} f\right\|_{2} .
$$

We then have the following:

Theorem 1.3. Suppose $0<\rho \leq 1 / 2,1<p \leq 3$, and $\nu>0$ is sufficiently small, depending only on $p$. Then for $\delta>0$ there exists $\epsilon>0$ depending only on $p, \delta$ such that if $\|\mathbf{b}\|_{3, p}<\epsilon$ and $\|f-A v f\|_{2, \nu} \leq \delta|A v f|$, one has $\left\|f_{\rho, \mathbf{b}}-A v f_{\rho, \mathbf{b}}\right\|_{2, \nu} \leq \delta\left|A v f_{\rho, \mathbf{b}}\right|$.

We consider the relationship between the proof of Theorem 1.1 and the proof of Theorem 1.2. Let $G_{D}$ be the Dirichlet Green's function for $-\Delta$ on $B_{1 / 4}$, whence $G_{D}$ is given explicitly by the formula,

$$
G_{D}(x, y)=\frac{1}{4 \pi|x-y|}-\frac{1}{16 \pi|y|} \frac{1}{|x-\bar{y}|},
$$

where $\bar{y}$ is the reflection of $y$ in $\partial B_{1 / 4}$. Let $T$ be the integral operator on functions with domain $B_{1 / 4}$ given by

$$
T f(x)=\int_{B_{1 / 4}} \mathbf{b}(x) \cdot \nabla_{x} G_{D}(x, y) f(y) d y, \quad x \in B_{1 / 4} .
$$


It was shown in $[\mathbf{C R}]$ that Theorem 1.1 is a consequence of the fact that $T$ is a bounded operator on the Morrey space $M_{r}^{q}$ with norm, $\|T\|$, satisfying $\|T\| \leq C\|\mathbf{b}\|_{3, p}$ for some constant $C$ depending only on $p, q, r$.

For $g$ a function with domain $\partial B_{1 / 4}$ let $v(y)=P g(y), y \in B_{1 / 4}$, be the solution of the Dirichlet problem,

$$
\begin{aligned}
\Delta v(y) & =0, \quad y \in B_{1 / 4}, \\
v(y) & =g(y), \quad y \in \partial B_{1 / 4} .
\end{aligned}
$$

The function $v$ is given explicitly by the Poisson formula,

$$
P g(y)=\frac{1}{\pi} \int_{\partial B_{1 / 4}} \frac{1 / 16-|y|^{2}}{|y-x|^{3}} g(x) d x, \quad y \in B_{1 / 4} .
$$

We can formally define an integral operator $Q$ on the functions $g$ by

$$
Q g(x)=\int_{B_{1 / 4}} d y G_{D}(x, y)(I-T)^{-1} \mathbf{b} \cdot \nabla P g(y), \quad x \in B_{1 / 4},
$$

where $T$ is given by (1.3). This operator induces an operator on functions with domain $S$ as follows: Let $f: S \rightarrow \mathbb{C}$ and denote also by $f$ the function with domain $\partial B_{1 / 4}$ naturally induced by $f$. Consider now the function $Q_{\rho} f$ with domain $\partial B_{(1-\rho) / 4}$ defined by

$$
Q_{\rho} f(x)=Q f(x), \quad x \in \partial B_{(1-\rho) / 4} .
$$

We can think of $Q_{\rho} f$ as a function with domain $S$, whence $Q_{\rho}$ is an operator on functions with domain $S$. In $[\mathbf{C O}]$ we proved that $Q_{\rho}$ is a bounded operator on $L^{2}(S), 0<\rho \leq 1 / 2$, with norm $\left\|Q_{\rho}\right\|$ satisfying $\left\|Q_{\rho}\right\| \leq C\|\mathbf{b}\|_{3, p}$ for some constant $C$ depending only on $\rho, p$, provided $\|\mathbf{b}\|_{3, p}<\epsilon$ for some $\epsilon$ depending only on $p$. Theorem 1.2 is a consequence of this fact.

Observe that the proof of Theorem 1.2 must be more difficult than the proof of Theorem 1.1 since in the definition of $Q_{\rho}$ one assumes that the inverse $(I-T)^{-1}$ exists. To prove Theorem 1.3 we need to know not only that $Q_{\rho}$ is bounded on $L^{2}(S)$ for $0<\rho \leq 1 / 2$ but also to have a bound which is uniform as $\rho \rightarrow 0$. Let $\langle$,$\rangle denote the scalar product on$ $L^{2}(S)$. In section 2 we prove the following: 
Theorem 1.4. Let $0<\rho \leq 1 / 2,1<p \leq 3$. Then there exists $C$, $\epsilon>0$ depending only on $p$, such that

$$
\left|\left\langle f, Q_{\rho} g\right\rangle\right| \leq C\|\mathbf{b}\|_{3, p}\|f\|_{2}\|g\|_{2},
$$

provided $\|\mathbf{b}\|_{3, p} \leq \epsilon, f, g \in L^{2}(S)$.

In order to prove Theorem 1.3 we need to know more detailed properties of $Q_{\rho}$ than those given in Theorem 1.4. In particular we must know that if $Q_{\rho}$ acts on a slowly varying function $g$ then the slowly varying component of $Q_{\rho} g$ has norm bounded linearly in $\rho$ as $\rho \rightarrow 0$. We also need to know that if $g$ is highly oscillatory then the slowly varying component of $Q_{\rho} g$ has small norm. These properties of $Q_{\rho}$ are summarised in the following:

Theorem 1.5. Let $0<\rho \leq 1 / 2,1<p \leq 3$. Then there exists $C, \epsilon$, $\eta>0$ depending only on $p$ such that $\|\mathbf{b}\|_{3, p}<\epsilon$ implies that

$$
\begin{array}{r}
\left|\left\langle E_{k^{\prime}} f, Q_{\rho} E_{k} g\right\rangle\right| \leq C\|\mathbf{b}\|_{3, p}\left\|E_{k^{\prime}} f\right\|_{2}\left\|E_{k} g\right\|_{2} \min \left[\rho 2^{k}, 1\right] \min \left[2^{\eta\left(k^{\prime}-k\right)}, 1\right] \\
0 \leq k, k^{\prime}<\infty
\end{array}
$$

We prove Theorem 1.5 in section 3 . Theorem 1.3 is a simple consequence of Theorems 1.4 and 1.5. It is proved in section 4 .

\section{Chapter 2. Configuration Space Localization}

To prove Theorem 1.4 we shall modify the proof in $[\mathbf{C O}]$ so that the estimates are uniform in $\rho$ as $\rho \rightarrow 0$.

Define an operator $A$ on functions $g$ with domain $S_{1 / 4}=\{|x|=1 / 4\}$ by

$$
A g(y)=|\mathbf{b}(y)|^{1 / 2} P g(y), \quad|y|<1 / 4
$$

Proposition 2.1. $A$ is a bounded linear operator from $L^{2}\left(S_{1 / 4}\right)$ to $L^{2}\left(B_{1 / 4}\right)$. There is a constant $C$ depending only on $p>1$ such that $\|A\| \leq C\|\mathbf{b}\|_{3, p}^{1 / 2}$. 
We shall prove Proposition 2.1 by following the general lines of the proof of Theorem 1.2 of [CR]. If a function $u$ is defined on the sphere $S_{1 / 4}$ then $A u$ is defined on the ball $B_{1 / 4}$. Let $Q_{0}$ be a cube with side of length 1 and for $n=1,2, \ldots$ let $Q_{n}$ be the dyadic subcubes of $Q_{0}$ with side of length $2^{-n}$. We define $u_{Q_{n}}$ as follows:

$$
\begin{array}{ll}
u_{Q_{n}}=0 & \text { if } Q_{n} \cap S_{1 / 4} \text { is empty, } \\
u_{Q_{n}}=\left|Q_{n}\right|^{-2 / 3} \int_{Q_{n} \cap S_{1 / 4}}|u(x)| d x, & \text { otherwise. }
\end{array}
$$

For $\xi \in \mathbb{R}^{3}$ let $Q_{0}(\xi)$ be the unit cube centered at $\xi$ with corresponding dyadic subcubes $Q_{n}(\xi)$. We then have the following:

Lemma 2.1. There exists a universal constant $C$ such that

$$
\begin{aligned}
& \int_{B_{1 / 4}}|A u(y)|^{2} d y \\
& \quad \leq C \int_{B_{1 / 4}} d \xi \sum_{n=0}^{\infty} \sum_{Q_{n}(\xi) \subset Q_{0}(\xi)} u_{Q_{n}(\xi)}^{2} \int_{Q_{n}(\xi)}|\mathbf{b}(y)| d y .
\end{aligned}
$$

Proof: We have from the definition (2.1) that

$$
A u(y)=|\mathbf{b}(y)|^{1 / 2} \frac{1}{16 \pi} \int_{S_{1 / 4}} \frac{1-16|y|^{2}}{|y-x|^{3}} u(x) d x .
$$

We estimate $A u(y)$ in the annulus

$$
R_{m}=\left\{y: \frac{1}{4}\left(1-2^{-m}\right) \leq|y|<\frac{1}{4}\left(1-2^{-(m+1)}\right)\right\}, \quad m=0,1,2, \ldots
$$

Thus

$$
|A u(y)| \leq C|\mathbf{b}(y)|^{1 / 2} 2^{-m} \sum_{n=0}^{m} 2^{3 n} \int_{S_{1 / 4} \cap\left\{|x-y|<2^{-n-1}\right\}}|u(x)| d x,
$$

for some universal constant $C$. Choosing $\alpha>0$ and applying the Schwarz inequality to the RHS of the previous expression we have

$$
\begin{aligned}
& |A u(y)|^{2} \\
& \leq C_{\alpha}|\mathbf{b}(y)| 2^{-2 m(1-\alpha)} \sum_{n=0}^{m} 2^{2(3-\alpha) n}\left[\int_{S_{1 / 4} \cap\left\{|x-y|<2^{-n-1}\right\}}|u(x)| d x\right]^{2},
\end{aligned}
$$


for some constant $C_{\alpha}$ depending only on $\alpha$. Thus

$$
\begin{aligned}
& \int_{B_{1 / 4}}|A u(y)|^{2} d y= \sum_{m=0}^{\infty} \int_{R_{m}}|A u(y)|^{2} d y \\
& \leq C_{\alpha} \sum_{0 \leq n \leq m<\infty} 2^{-2 m(1-\alpha)} 2^{2(3-\alpha) n} \int_{R_{m}} d y|\mathbf{b}(y)| \\
& {\left[\int_{S_{1 / 4} \cap\left\{|x-y|<2^{-n-1}\right\}}|u(x)| d x\right]^{2} . }
\end{aligned}
$$

Let $U_{n}=\cup_{m=n}^{\infty} R_{m}, n \geq 0$. Then it is clear from the previous expression that if $\alpha<1$ there is a constant $C_{\alpha}$ depending only on $\alpha$ such that

$$
\int_{B_{1 / 4}}|A u(y)|^{2} d y \leq C_{\alpha} \sum_{n=0}^{\infty} a_{n},
$$

where

$$
a_{n}=\int_{U_{n}} d y|\mathbf{b}(y)|\left[2^{2 n} \int_{S_{1 / 4} \cap\left\{|x-y|<2^{-n-1}\right\}}|u(x)| d x\right]^{2} .
$$

It is clear now that there exists a universal constant $C$ such that

$$
a_{n} \leq C \int_{B_{1 / 4}} d \xi \sum_{Q_{n}(\xi) \subset Q_{0}(\xi)} u_{Q_{n}}^{2}(\xi) \int_{Q_{n}(\xi)}|\mathbf{b}(y)| d y, \quad n \geq 0 .
$$

The result follows then from the last two inequalities.

Next we shall show that for fixed $\xi$ the RHS of (2.2) is bounded by the $L^{2}$ norm of $u$.

Lemma 2.2. Let $Q_{0}$ be a cube in $\mathbb{R}^{3}$ with side of length 1 and dyadic subcubes $Q_{n}$ with side of length $2^{-n}, n=1,2, \ldots$. Then there is a constant $C$ depending only on $p>1$ such that

$$
\sum_{n=0}^{\infty} \sum_{Q_{n} \subset Q_{0}} u_{Q_{n}}^{2} \int_{Q_{n}}|\mathbf{b}(y)| d y \leq C\|\mathbf{b}\|_{3, p}\|u\|_{2}^{2} .
$$

Evidently Proposition 2.1 follows from Lemma 2.1 and Lemma 2.2. Observe that for fixed $n$ one has

$$
\begin{aligned}
\sum_{Q_{n} \subset Q_{0}} u_{Q_{n}}^{2} \int_{Q_{n}}|\mathbf{b}(y)| d y & \leq \sum_{Q_{n} \subset Q_{0}} u_{Q_{n}}^{2}\|\mathbf{b}\|_{3, p} 2^{-2 n} \\
& \leq C\|\mathbf{b}\|_{3, p} \sum_{Q_{n} \subset Q_{0}} \int_{Q_{n} \cap S_{1 / 4}}|u(x)|^{2} d x \\
& \leq C\|\mathbf{b}\|_{3, p}\|u\|_{2}^{2},
\end{aligned}
$$


for some universal constant $C$. In order to do the summation with respect to $n$ in (2.3) we need to resort to a Calderon-Zygmund decomposition. First we have

Lemma 2.3. Let $Q^{\prime}$ be a cube in $\mathbb{R}^{3}$ with side of length $2^{-n_{Q^{\prime}}}$, where $n_{Q^{\prime}}$ is a nonnegative integer. Suppose for some $\varepsilon>0$ one has $|Q|^{\varepsilon} u_{Q} \leq$ $\left|Q^{\prime}\right|^{\varepsilon} u_{Q^{\prime}}$ for all dyadic subcubes $Q$ of $Q^{\prime}$. Then if $\varepsilon$ is sufficiently small there exists a constant $C$ depending only on $p>1$ such that

$$
\sum_{Q \subset Q^{\prime}} u_{Q}^{2} \int_{Q}|\mathbf{b}(y)| d y \leq C\|\mathbf{b}\|_{3, p}\left|Q^{\prime}\right|^{2 / 3} u_{Q^{\prime}}^{2}
$$

Proof: We have for fixed $n \geq n_{Q^{\prime}}$,

$$
\begin{aligned}
\sum_{Q_{n} \subset Q^{\prime}} & u_{Q_{n}}^{2} \int_{Q_{n}}|\mathbf{b}(y)| d y \\
& \leq 2^{6\left(n-n_{Q^{\prime}}\right) \varepsilon} u_{Q^{\prime}}^{2} \sum_{\left\{Q_{n}: Q_{n} \subset Q^{\prime}, Q_{n} \cap S_{1 / 4} \neq \emptyset\right\}} \int_{Q_{n}}|\mathbf{b}(y)| d y \\
& \leq 2^{6\left(n-n_{Q^{\prime}}\right) \varepsilon} u_{Q^{\prime}}^{2} \int_{Q^{\prime} \cap\left\{y: 1>|y|>1-2^{-n} \sqrt{3}\right\}}|\mathbf{b}(y)| d y \\
& \leq 2^{6\left(n-n_{Q^{\prime}}\right) \varepsilon} u_{Q^{\prime}}^{2} \operatorname{meas}\left(Q^{\prime} \cap\left\{y: 1>|y|>1-2^{-n \sqrt{3}\}}\right)^{1-1 / p}\right. \\
& \left.\leq\left. C 2^{6\left(n-n_{Q^{\prime}}\right) \varepsilon} u_{Q^{\prime}}^{2}\left(2^{-2 n_{Q^{\prime}} 2^{-n}}\right)^{1-1 / p}\|\mathbf{b}\|_{3, p}\left|Q^{\prime}\right|^{1 / p-1 / 3}\left|\int_{Q^{\prime}}\right| \mathbf{b}(y)\right|^{p} d y\right]^{1 / p} \\
& =C\|\mathbf{b}\|_{3, p}\left|Q^{\prime}\right|^{2 / 3} u_{Q^{\prime}}^{2} 2^{\left(n-n_{Q^{\prime}}\right)(6 \varepsilon+1 / p-1)},
\end{aligned}
$$

where $C$ is a universal constant.

If we now choose $\varepsilon$ to satisfy $\varepsilon<(1-1 / p) / 6$, then we have

$$
\begin{aligned}
\sum_{Q \subset Q^{\prime}} u_{Q}^{2} \int_{Q}|\mathbf{b}(y)| d y & \leq C\|\mathbf{b}\|_{3, p}\left|Q^{\prime}\right|^{2 / 3} u_{Q^{\prime}}^{2} \sum_{n=n_{Q^{\prime}}}^{\infty} 2^{\left(n-n_{Q^{\prime}}\right)(6 \varepsilon+1 / p-1)} \\
& \leq C_{p}\|\mathbf{b}\|_{3, p}\left|Q^{\prime}\right|^{2 / 3} u_{Q^{\prime}}^{2}
\end{aligned}
$$

where the constant $C_{p}$ depends only on $p>1$. 
Let $Q_{0}$ be a unit cube in $\mathbb{R}^{3}$. We make a Calderon-Zygmund decomposition of $Q_{0}$ based on the criterion in Lemma 2.3. In particular we define a sequence of families $\mathcal{F}_{j}$ of dyadic subcubes of $Q_{0}, j=0,1,2, \ldots$ as follows: $\mathcal{F}_{0}=\left\{Q_{0}\right\}$. Let $G_{1} \subset Q_{0}$ be defined as

$$
G_{1}=\left\{x \in Q_{0}:|Q|^{\varepsilon} u_{Q} \leq\left|Q_{0}\right|^{\varepsilon} u_{Q_{0}}\right.
$$

for all dyadic subcubes $Q$ of $Q_{0}$ with $\left.x \in Q\right\}$.

Then there is a unique finite family $\mathcal{F}_{1}$ of disjoint dyadic subcubes of $Q_{0}$ such that

$$
\bigcup_{Q \in \mathcal{F}_{1}} Q=Q_{0} \backslash G_{1}
$$

Proceeding by induction as in section 2 of $[\mathbf{C R}]$, we can construct sets $G_{j}$ and families $\mathcal{F}_{j}, j \geq 1$, with the properties

(a) $\bigcup_{j=1}^{\infty} G_{j}=Q_{0}$.

(b) $\bigcup_{Q \in \mathcal{F}_{k}} Q=Q_{0} \backslash \bigcup_{j=1}^{k} G_{j}$.

(c) For any $Q \in \mathcal{F}_{k}$ let $\bar{Q} \in \mathcal{F}_{k-1}$ be the unique dyadic subcube containing $Q$. Then

$$
|Q|^{\varepsilon} u_{Q}>|\bar{Q}|^{\varepsilon} u_{\bar{Q}}
$$

It is clear now from Lemma 2.3 that there is a constant $C$ depending only on $p>1$ such that

$$
\sum_{n=0}^{\infty} \sum_{Q_{n} \subset Q_{0}} u_{Q_{n}}^{2} \int_{Q_{n}}|\mathbf{b}(y)| d y \leq C\|\mathbf{b}\|_{3, p} \sum_{j=0}^{\infty} \sum_{Q \in \mathcal{F}_{j}}|Q|^{2 / 3} u_{Q}^{2} .
$$

The proof of Lemma 2.2 will be complete if we can show

Lemma 2.4. There exists a constant $C$ depending only on $p>1$ such that

$$
\sum_{j=0}^{\infty} \sum_{Q \in \mathcal{F}_{j}}|Q|^{2 / 3} u_{Q}^{2} \leq C\|u\|_{2}^{2}
$$


Proof: We shall assume without lost of generality that $\|u\|_{\infty}<\infty$. Hence there exists an integer $t \geq 1$ such that $\mathcal{F}_{t}$ is empty, whence

$$
Q_{0}=\bigcup_{j=1}^{t} G_{j} .
$$

Let us consider a particular $Q \in \mathcal{F}_{j}, 0 \leq j \leq t-1$. It is evident that

$$
Q \subset \bigcup_{m=j+1}^{t} G_{m}
$$

We wish to estimate the ratio $\left|Q \cap G_{m} \cap S_{1 / 4}\right| /|Q|^{2 / 3}$ for $m \geq j+1$. We have now

$$
\begin{aligned}
|Q|^{2 / 3} u_{Q} & =\int_{Q \cap S_{1 / 4}}|u(x)| d x \geq \sum_{i=m}^{t} \int_{Q \cap G_{i} \cap S_{1 / 4}}|u| d x \\
& =\sum_{\bar{Q} \in \mathcal{F}_{m-1}, \bar{Q} \subset Q}|\bar{Q}|^{2 / 3} u_{\bar{Q}} \geq \sum_{\bar{Q} \in \mathcal{F}_{m-1}, \bar{Q} \subset Q}|\bar{Q}|^{2 / 3}\left(\frac{|Q|}{|\bar{Q}|}\right)^{\varepsilon} u_{Q} \\
& \geq 2^{3(m-j-1) \varepsilon} u_{Q} \sum_{\bar{Q} \in \mathcal{F}_{m-1}, \bar{Q} \subset Q}|\bar{Q}|^{2 / 3} \\
& \geq c 2^{3(m-j-1) \varepsilon} u_{Q}\left|Q \cap \bigcup_{i=m}^{t} G_{i} \cap S_{1 / 4}\right|
\end{aligned}
$$

for some universal constant $c>0$. We conclude therefore that

$$
\left|Q \cap G_{m} \cap S_{1 / 4}\right| /|Q|^{2 / 3} \leq c^{-1} 2^{-3(m-j-1) \varepsilon} .
$$

Next we write

$$
\begin{aligned}
|Q|^{2 / 3} u_{Q}^{2} & =|Q|^{-2 / 3}\left[\int_{Q \cap S_{1 / 4}}|u| d x\right]^{2} \\
& =|Q|^{-2 / 3}\left[\sum_{m=j+1}^{t} \int_{Q \cap G_{m} \cap S_{1 / 4}}|u| d x\right]^{2} \\
& \leq|Q|^{-2 / 3}\left[\sum_{m=j+1}^{t} a_{m}^{2}\right] \sum_{m=j+1}^{t} a_{m}^{-2}\left[\int_{Q \cap G_{m} \cap S_{1 / 4}}|u| d x\right]^{2}
\end{aligned}
$$


for any positive sequence $a_{m}$. We choose $a_{m}$ to be given by

$$
a_{m}=\left[(3 / 2)^{3(m-j-1) \varepsilon}\left|Q \cap G_{m} \cap S_{1 / 4}\right| /|Q|^{2 / 3}\right]^{1 / 2} .
$$

Then, in view of the last two inequalities we have

$|Q|^{2 / 3} u_{Q}^{2} \leq C \sum_{m=j+1}^{t}\left(\frac{2}{3}\right)^{3(m-j-1) \varepsilon}\left|Q \cap G_{m} \cap S_{1 / 4}\right|^{-1}\left[\int_{Q \cap G_{m} \cap S_{1 / 4}}|u| d x\right]^{2}$,

for some constant $C$ depending only on $\varepsilon>0$. We conclude then that

$$
\sum_{Q \in \mathcal{F}_{j}}|Q|^{2 / 3} u_{Q}^{2} \leq C \sum_{m=j+1}^{t}\left(\frac{2}{3}\right)^{3(m-j-1) \varepsilon} \int_{G_{m} \cap S_{1 / 4}}|u|^{2} d x .
$$

Now if we sum this last inequality with respect to $j$ and use the fact that the sets $G_{j}$ are disjoint we obtain the inequality (2.4).

We return now to the operator $Q_{\rho}$ of (1.6). The proof in section 4 of [CO] that $Q_{\rho}$ is a bounded operator on $L^{2}$ had three ingredients. The first stage was to show that the function $\mathbf{b} \cdot \nabla \mathrm{Pg}$ with domain $B_{1 / 4}$ is in an appropriate Morrey space, This Morrey space, $M_{r}^{3 / 2}\left(B_{1 / 4}\right)$, is defined to be the set of functions $h: B_{1 / 4} \rightarrow \mathbb{R}$ such that

$$
\int_{Q \cap B_{1 / 4}}(1 / 4-|y|)^{r}|h(y)|^{r} d y \leq C^{r}|Q|^{1-2 r / 3},
$$

on all cubes $Q$. The norm of $h,\|h\|_{3 / 2, r}$, is the minimum $C$ satisfying the previous inequality. It was shown in $[\mathbf{C O}]$ that $\mathbf{b} \cdot \nabla P g$ is in $M_{r}^{3 / 2}\left(B_{1 / 4}\right)$ provided $r$ satisfies $r /(2-r)<p$. We see now how this follows from Proposition 2.1. By the Harnack inequality there is a constant $C$ such that

$$
\begin{aligned}
& (1 / 4-|y|)|\mathbf{b}(y) \cdot \nabla P g(y)| \leq C|\mathbf{b}(y)||P g(y)| \\
& =C|\mathbf{b}(y)|^{1 / 2}|A g(y)|, \quad|y|<1 / 4 .
\end{aligned}
$$

Hence for any cube $Q$ one has

$$
\begin{aligned}
\int_{Q \cap B_{1 / 4}} & (1 / 4-|y|)^{r}|\mathbf{b}(y) \cdot \nabla P g(y)|^{r} d y \\
& \leq C\left[\int_{B_{1 / 4}}|A g(y)|^{2} d y\right]^{r / 2}\left[\int_{Q}|\mathbf{b}(y)|^{r /(2-r)} d y\right]^{1-r / 2} \\
& \leq C\|A\|^{r}\|g\|_{2}^{r}\|\mathbf{b}\|_{3, p}^{r / 2}|Q|^{1-2 r / 3} \\
& \leq C_{1}\|g\|_{2}^{r}\|\mathbf{b}\|_{3, p}^{r}|Q|^{1-2 r / 3}
\end{aligned}
$$


If follows that if $r /(2-r)<p$ the function $\mathbf{b} \cdot \nabla P g$ is in $M_{r}^{3 / 2}\left(B_{1 / 4}\right)$ and

$$
\|\mathbf{b} \cdot \nabla P g\|_{3 / 2, r} \leq C\|\mathbf{b}\|_{3, p}\|g\|_{2}
$$

for some constant $C$ depending only on $p>1$.

The second stage was to prove that, for $T$ the integral operator given by (1.3), the function $(I-T)^{-1} \mathbf{b} \cdot \nabla P g$ is also in $M_{r}^{3 / 2}\left(B_{1 / 4}\right)$. Thus the perturbation due to the integral operator $T$ is small in the sense that it preserves the space in which $\mathbf{b} \cdot \nabla P g$ lies. We shall do something analagous here. For $y \in \mathbb{R}^{3}$ let us define a vector $\mathbf{n}(y)$ by

$$
\begin{aligned}
\mathbf{n}(y) & =0 & & \text { if } \mathbf{b}(y)=0 . \\
& =\mathbf{b}(y) /|\mathbf{b}(y)| & & \text { otherwise. }
\end{aligned}
$$

Evidently one has $|\mathbf{n}(y)| \leq 1$ for all $y \in \mathbb{R}^{3}$. Let $T_{\text {sym }}$ be the integral operator on functions with domain $B_{1 / 4}$ which has kernel

$$
|\mathbf{b}(x)|^{1 / 2} \mathbf{n}(x) \cdot \nabla_{x} G_{D}(x, y)|\mathbf{b}(y)|^{1 / 2}, \quad x, y \in B_{1 / 4}
$$

Then we may formally write

$$
(I-T)^{-1} \mathbf{b} \cdot \nabla P g(y)=|\mathbf{b}(y)|^{1 / 2}\left(I-T_{\mathrm{sym}}\right)^{-1}|\mathbf{b}|^{1 / 2} \mathbf{n} \cdot \nabla P g(y), \quad y \in B_{1 / 4}
$$

Let $L_{\text {weight }}^{2}\left(B_{1 / 4}\right)$ be the weighted $L^{2}$ space of functions $h: B_{1 / 4} \rightarrow \mathbb{C}$ such that

$$
\|h\|_{2, \text { weight }}^{2}=\int_{B_{1 / 4}}(1 / 4-|y|)^{2}|h(y)|^{2} d y<\infty .
$$

From Proposition 2.1 and Harnack the function $|\mathbf{b}|^{1 / 2} \mathbf{n} \cdot \nabla P g$ is in this space.

Proposition 2.2. The operator $T_{\mathrm{sym}}$ is bounded on the space $L_{\text {weight }}^{2}\left(B_{1 / 4}\right)$ and there exists a constant $C>0$ depending only on $p>1$ such that

$$
\left\|T_{\text {sym }}\right\| \leq C\|\mathbf{b}\|_{3, p}
$$

Remark 2.1. It is interesting to compute the information that the results of Olsen $[\mathbf{O}]$ give about the operator $T_{\text {sym }}$. If we apply Theorem 2 of $[\mathbf{O}]$ then

$$
\left|T_{\mathrm{sym}} h(x)\right| \leq T_{g} f(x), \quad x \in B_{1 / 4},
$$


where $f(y)=|\mathbf{b}(y)|^{1 / 2}|h(y)|$ and $g(x)=|\mathbf{b}(x)|^{1 / 2} / 2 \pi$. Then if we assume $h$ is in $L^{2}\left(B_{1 / 4}\right)$ we have in the notation of Theorem 2 of $[\mathbf{O}] p=3 / 2$, $r=3 / 2, v=6$, and $u, q$ can be taken slightly larger than 2,1 respectively. Hence $T_{g} f \in M_{s}^{t}$ where

$$
\begin{aligned}
& \frac{1}{t}=\frac{1}{v}+\frac{1}{r}+\frac{1}{p}-1=\frac{1}{6}+\frac{2}{3}+\frac{2}{3}-1=\frac{1}{2} \\
& \frac{1}{s}=\frac{1}{v}+\frac{1}{q}+\frac{1}{p}-1=\frac{1}{6}+\frac{1}{q}+\frac{2}{3}-1=\frac{1}{q}-\frac{1}{6} .
\end{aligned}
$$

Now if $s=2$ then $T_{\text {sym }} h$ is also in $L^{2}\left(B_{1 / 4}\right)$. Evidently $s=2$ implies $q=3 / 2$ which implies $q /(2-q)=3$ whence we need to have $\mathbf{b} \in L^{3}\left(\mathbb{R}^{3}\right)$. We can do better than this by combining various theorems of $[\mathbf{O}]$.

Proposition 2.3. The operator $T_{\mathrm{sym}}$ is bounded on the space $L^{2}\left(B_{1 / 4}\right)$ and there exists a constant $C>0$ depending only on $p>1$ such that

$$
\left\|T_{\mathrm{sym}}\right\| \leq C\|\mathbf{b}\|_{3, p}
$$

Proof: We have

$$
\begin{aligned}
\left|T_{\mathrm{sym}} h(x)\right| & \leq \frac{|\mathbf{b}(x)|^{1 / 2}}{2 \pi} \int_{B_{1 / 4}} \frac{|\mathbf{b}(y)|^{1 / 2}|h(y)|}{|x-y|^{2}} d y \\
& \leq \frac{|\mathbf{b}(x)|^{1 / 2}}{2 \pi}\left[\int_{B_{1 / 4}} \frac{|\mathbf{b}(x)|^{q^{\prime} / 2}}{|x-y|^{2}} d y\right]^{1 / q^{\prime}}\left[\int_{B_{1 / 4}} \frac{|h(y)|^{q}}{|x-y|^{2}} d y\right]^{1 / q},
\end{aligned}
$$

where $1 / q+1 / q^{\prime}=1$. We choose $q^{\prime}$ in the range $2<q^{\prime}<2 p$ whence $q<2$. Consider now the function $g_{1}(x)$ defined by

$$
g_{1}(x)=\int_{B_{1 / 4}} \frac{|\mathbf{b}(x)|^{q^{\prime} / 2}}{|x-y|^{2}} d y .
$$

Now $|\mathbf{b}|^{q^{\prime} / 2}$ is in the Morrey space $M_{2 p / q^{\prime}}^{6 / q^{\prime}}$. Hence by Theorem 9 of $[\mathbf{O}]$ $g_{1}$ is in $M_{s}^{t}$ where

$$
1 / t=q^{\prime} / 6-1 / 3, \quad s=t\left(2 p / q^{\prime}\right) /\left(6 / q^{\prime}\right)=t p / 3,
$$

and $\left\|g_{1}\right\|_{t, s} \leq C\|\mathbf{b}\|_{3, p}^{q^{\prime} / 2}$ for some constant $C$ depending only on $p, q^{\prime}$. Next let $g(x)$ be given by

$$
g(x)=\frac{|\mathbf{b}(x)|^{q / 2}}{(2 \pi)^{q}} g_{1}(x)^{q / q^{\prime}} .
$$


Then

$$
\left|T_{\mathrm{sym}} h(x)\right| \leq\left[T_{g}|h|^{q}(x)\right]^{1 / q}
$$

By Holder's inequality for Morrey spaces, Lemma 11 of $[\mathbf{O}]$, we have that $g$ is in $M_{p}^{3}$ since

$$
\begin{aligned}
q / 6+q / q^{\prime} t & =q / 6+\left(q / q^{\prime}\right)\left[q^{\prime} / 6-1 / 3\right]=1 / 3, \\
q / 2 p+q / q^{\prime} s & =q / 2 p+\left(q / q^{\prime}\right)(3 / p)\left[q^{\prime} / 6-1 / 3\right]=1 / p .
\end{aligned}
$$

Furthermore,

$$
\|g\|_{3, p} \leq\|\mathbf{b}\|_{3, p}^{q / 2}\left\|g_{1}\right\|_{t, s}^{q / q^{\prime}} \leq C^{q-1}\|\mathbf{b}\|_{3, p}^{q}
$$

Now $|h|^{q}$ is in the space $L^{2 / q}\left(B_{1 / 4}\right)$. Hence by Corollary 3 of $[\mathbf{O}] T_{g}|h|^{q}$ is also in the space $L^{2 / q}\left(B_{1 / 4}\right)$ provided $2 / q<p$. Furthermore

$$
\left\|T_{g}|h|^{q}\right\|_{2 / q} \leq C_{1}\|g\|_{3, p}\left\||h|^{q}\right\|_{2 / q} \leq C_{1} C^{q-1}\|\mathbf{b}\|_{3, p}^{q}\|h\|_{2}^{q},
$$

for some constant $C_{1}$ depending only on $q, p$. Now from (2.5) and the previous inequality we conclude that

$$
\left\|T_{\text {sym }} h\right\|_{2} \leq C_{1}^{1 / q} C^{1 / q^{\prime}}\|\mathbf{b}\|_{3, p}\|h\|_{2}
$$

The result follows since if $p>1$ the two inequalities $2<q^{\prime}<2 p, 2 / q<p$ can be simultaneously satisfied. In fact $q$ can be chosen in the range

$$
\frac{1}{2}<\frac{1}{q}<\min [p / 2,1-1 / 2 p]=1-1 / 2 p .
$$

Remark 2.2. Theorem 9 of $[\mathbf{O}]$ was originally proved in $[\mathbf{A}]$. A different proof was given in $[\mathbf{C F}]$.

The proof of Proposition 2.3 gives us an important insight into how the proof of Proposition 2.2 should go. We shall follow the lines of the proof of Theorem 1.2 of $[\mathbf{C R}]$ and Proposition 2.1 of $[\mathbf{C O}]$, but the CalderonZygmund decomposition will be based on taking averages of $|h(y)|^{q}$. Let $Q_{0}$ be a unit cube and for $n=1,2, \ldots Q_{n}$ be the dyadic subcubes of $Q_{0}$ with side of length $2^{-n}$. Let $d\left(Q_{n}\right)$ be given by

$$
d\left(Q_{n}\right)=\sup \left\{d\left(x, \partial B_{1 / 4}\right): x \in Q_{n}\right\} .
$$


For $n=0,1,2, \ldots$ define an operator $S_{n}$ on functions $h$ with domain $B_{1 / 4}$ by

$S_{n} h(x)=\frac{2^{2 n}}{d\left(Q_{n}\right)} \int_{Q_{n} \cap B_{1 / 4}}(1 / 4-|y|)|\mathbf{b}(y)|^{1 / 2}|h(y)| d y, \quad x \in Q_{n} \cap B_{1 / 4}$.

Let $T_{Q_{0}}$ be the operator given by

$$
T_{Q_{0}} h(x)=\sum_{n=0}^{\infty}|\mathbf{b}(x)|^{1 / 2} S_{n} h(x), \quad x \in B_{1 / 4}
$$

Then Jensen's inequality implies there is a universal constant $C$ such that if $Q_{0}(\xi)$ is the unit cube centered at $\xi$,

$$
\begin{aligned}
\int_{B_{1 / 4}}(1 / 4 & -|x|)^{2}\left|T_{\mathrm{sym}} h(x)\right|^{2} d x \\
& \leq C \int_{B_{1 / 4}} d \xi \int_{Q_{0}(\xi) \cap B_{1 / 4}}(1 / 4-|x|)^{2}\left|T_{Q_{0}(\xi)} h(x)\right|^{2} d x .
\end{aligned}
$$

Hence it is sufficient to prove Proposition 2.2 with the operator $T_{\text {sym }}$ replaced by $T_{Q_{0}}$. The key lemma analagous to Lemma 2.4 of $[\mathbf{C R}]$ is

Lemma 2.5. Let $Q^{\prime} \subset Q_{0}$ be an arbitrary dyadic subcube of $Q_{0}$ with side of length $2^{-n_{Q^{\prime}}}$. Suppose $1<p \leq 3$ and $q>1$ satisfies the inequality $1 / 2<1 / q<1-1 / 2 p$. For $Q$ a dyadic subcube of $Q_{0}$ and $h$ a function with domain $B_{1 / 4}$ let $h_{Q}$ be given by

$$
h_{Q}=\left[\frac{1}{|Q|} \int_{Q \cap B_{1 / 4}}(1 / 4-|y|)^{q}|h(y)|^{q} d y\right]^{1 / q} .
$$

Then there are constants $C, \varepsilon>0$ depending only on $p$ and $q$ such that

$$
|Q|^{1 / 6+\varepsilon} h_{Q} \leq\left|Q^{\prime}\right|^{1 / 6+\varepsilon} h_{Q^{\prime}}
$$

for all dyadic subcubes $Q$ of $Q^{\prime}$ implies the inequality

$$
\int_{Q^{\prime} \cap B_{1 / 4}}(1 / 4-|x|)^{2}\left[\sum_{n=n_{Q^{\prime}}}^{\infty}|\mathbf{b}(x)|^{1 / 2} S_{n} h(x)\right]^{2} d x \leq C^{2}\|\mathbf{b}\|_{3, p}^{2}\left|Q^{\prime}\right| h_{Q^{\prime}}^{2} .
$$


Proof: We have

$$
\left[\sum_{n=n_{Q^{\prime}}}^{\infty} S_{n} h(x)\right]^{2} \leq 2 \sum_{k=n_{Q^{\prime}}}^{\infty} S_{k} h(x) \sum_{n=n_{Q^{\prime}}}^{k} S_{n} h(x) .
$$

It follows from Holder's inequality that

$$
S_{n} h(x) \leq \frac{2^{2 n}}{d\left(Q_{n}\right)}\left[\int_{Q_{n} \cap B_{1 / 4}}(1 / 4-|y|)^{q}|h(y)|^{q} d y\right]^{1 / q}\left[\int_{Q_{n}}|\mathbf{b}(y)|^{q^{\prime} / 2} d y\right]^{1 / q^{\prime}}
$$

where $1 / q+1 / q^{\prime}=1$. Since $1 / q<1-1 / 2 p$ implies $q^{\prime} / 2<p$ we conclude that

$$
S_{n} h(x) \leq\|\mathbf{b}\|_{3, p}^{1 / 2}\left|Q_{n}\right|^{1 / 6} h_{Q_{n}} / d\left(Q_{n}\right), \quad x \in Q_{n},
$$

whence

$$
(1 / 4-|x|) S_{n} h(x) \leq\|\mathbf{b}\|_{3, p}^{1 / 2}\left|Q_{n}\right|^{1 / 6} h_{Q_{n}}, \quad x \in Q_{n}
$$

Now if we use (2.7) we have from the previous inequality

$$
\begin{aligned}
(1 / 4-|x|) \sum_{n=n_{Q^{\prime}}}^{k} S_{n} h(x) & \leq\|\mathbf{b}\|_{3, p}^{1 / 2} \sum_{n=n_{Q^{\prime}}}^{k}\left|Q_{n}\right|^{1 / 6} h_{Q_{n}} \\
& \leq\|\mathbf{b}\|_{3, p}^{1 / 2}\left|Q^{\prime}\right|^{1 / 6} h_{Q^{\prime}} \sum_{n=n_{Q^{\prime}}}^{k} 2^{3 \varepsilon\left(n-n_{Q^{\prime}}\right)} \\
& \leq C_{\varepsilon}\|\mathbf{b}\|_{3, p}^{1 / 2}\left|Q^{\prime}\right|^{1 / 6} h_{Q^{\prime}} 2^{3 \varepsilon\left(k-n_{Q^{\prime}}\right)}
\end{aligned}
$$

for some constant $C_{\varepsilon}$ depending only on $\varepsilon>0$. Hence

$$
\begin{aligned}
& \text { (2.9) } \int_{Q^{\prime} \cap B_{1 / 4}}(1 / 4-|x|)^{2}\left[\sum_{n=n_{Q^{\prime}}}^{\infty}|\mathbf{b}(x)|^{1 / 2} S_{n} h(x)\right]^{2} d x \\
& \leq 2 C_{\varepsilon}\|\mathbf{b}\|_{3, p}^{1 / 2}\left|Q^{\prime}\right|^{1 / 6} h_{Q^{\prime}} \sum_{k=n_{Q^{\prime}}}^{\infty} 2^{3 \varepsilon\left(k-n_{Q^{\prime}}\right)} \int_{Q^{\prime} \cap B_{1 / 4}}(1 / 4-|x|)|\mathbf{b}(x)| S_{k} h(x) d x .
\end{aligned}
$$

For $m$ an integer let $E_{m}$ be the set

$$
E_{m}=\left\{x \in \mathbb{R}^{3}: 2^{m-1}<|\mathbf{b}(x)| \leq 2^{m}\right\} .
$$


For $m, k$ integers and $k \geq n_{Q^{\prime}}$ let $a_{m, k}$ be given by

$$
a_{m, k}=\sum_{Q_{k} \subset Q^{\prime}}\left|E_{m} \cap Q_{k}\right| \int_{Q_{k}}(1 / 4-|y|)|\mathbf{b}(y)|^{1 / 2}|h(y)| d y .
$$

Then we have

$$
\begin{aligned}
\sum_{k=n_{Q^{\prime}}}^{\infty} 2^{3 \varepsilon\left(k-n_{Q^{\prime}}\right)} \int_{Q^{\prime} \cap B_{1 / 4}} & (1 / 4-|x|)|\mathbf{b}(x)| S_{k} h(x) d x \\
\leq & \sum_{m=-\infty}^{\infty} \sum_{k=n_{Q^{\prime}}}^{\infty} 2^{3 \varepsilon\left(k-n_{Q^{\prime}}\right)} 2^{m+2 k} a_{m, k}
\end{aligned}
$$

There are two estimates on $a_{m, k}$ which we use. The first follows from (2.7). Thus from (2.8) we have

$$
a_{m, k} \leq \sum_{Q_{k} \subset Q^{\prime}}\left|E_{m} \cap Q_{k}\right|\|\mathbf{b}\|_{3, p}^{1 / 2} 2^{-5 k / 2} h_{Q_{k}}
$$

and then (2.7) implies that

$$
a_{m, k} \leq\left|E_{m} \cap Q^{\prime}\right|\|\mathbf{b}\|_{3, p}^{1 / 2} 2^{-5 k / 2} 2^{(1 / 2+3 \varepsilon)\left(k-n_{Q^{\prime}}\right)} h_{Q^{\prime}} .
$$

The second estimate is obtained by using the fact that $\left|E_{m} \cap Q_{k}\right| \leq\left|Q_{k}\right|$.

Thus

$$
a_{m, k} \leq 2^{-3 k} \int_{Q^{\prime}}(1 / 4-|y|)|\mathbf{b}(y)|^{1 / 2}|h(y)| d y .
$$

If we again apply (2.8) we have that

$$
a_{m, k} \leq 2^{-3 k}\|\mathbf{b}\|_{3, p}^{1 / 2}\left|Q^{\prime}\right|^{5 / 6} h_{Q^{\prime}} .
$$

If follows now that for any $\alpha, 0<\alpha<1$, we have

$$
\begin{aligned}
\sum_{m=-\infty}^{\infty} \sum_{k=n_{Q^{\prime}}}^{\infty} 2^{3 \varepsilon\left(k-n_{Q^{\prime}}\right)} 2^{m+2 k} a_{m, k} & \\
\leq & \sum_{m=-\infty}^{\infty} \sum_{k=n_{Q^{\prime}}}^{\infty} 2^{3 \varepsilon\left(k-n_{Q^{\prime}}\right)} 2^{m+2 k}\left[2^{-3 k}\|\mathbf{b}\|_{3, p}^{1 / 2}\left|Q^{\prime}\right|^{5 / 6} h_{Q^{\prime}}\right]^{\alpha} \\
& \quad\left[\left|E_{m} \cap Q^{\prime}\right|\|\mathbf{b}\|_{3, p}^{1 / 2} 2^{-5 k / 2} 2^{(1 / 2+3 \varepsilon)\left(k-n_{Q^{\prime}}\right)} h_{Q^{\prime}}\right]^{1-\alpha} .
\end{aligned}
$$


For any $\alpha>0$ one can find sufficiently small $\varepsilon>0$ such that the sum with respect to $k$ above converges. Thus there is a constant $C_{\alpha}>0$ depending only on $\alpha, \varepsilon$ and

$$
\begin{aligned}
& \sum_{m=-\infty}^{\infty} \sum_{k=n_{Q^{\prime}}}^{\infty} 2^{3 \varepsilon\left(k-n_{Q^{\prime}}\right)} 2^{m+2 k} a_{m, k} \\
& \leq C_{\alpha}\|\mathbf{b}\|_{3, p}^{1 / 2}\left|Q^{\prime}\right|^{\alpha+1 / 6} h_{Q^{\prime}} \sum_{m=-\infty}^{\infty} 2^{m}\left|E_{m} \cap Q^{\prime}\right|^{1-\alpha} .
\end{aligned}
$$

Let $m_{0}$ be an arbitrary integer. Evidently one has

$$
\sum_{m=-\infty}^{m_{0}} 2^{m}\left|E_{m} \cap Q^{\prime}\right|^{1-\alpha} \leq\left|Q^{\prime}\right|^{1-\alpha} \sum_{m=-\infty}^{m_{0}} 2^{m}=2\left|Q^{\prime}\right|^{1-\alpha} 2^{m_{0}} .
$$

Using the fact that

$$
2^{m p}\left|E_{m} \cap Q^{\prime}\right| \leq 2^{p}\|\mathbf{b}\|_{3, p}^{p}\left|Q^{\prime}\right|^{1-p / 3},
$$

it follows that if $\alpha>0$ satisfies $(1-\alpha) p>1$ then

$$
\sum_{m=m_{0}+1}^{\infty} 2^{m}\left|E_{m} \cap Q^{\prime}\right|^{1-\alpha} \leq C_{\alpha}\|\mathbf{b}\|_{3, p}^{p(1-\alpha)}\left|Q^{\prime}\right|^{(1-p / 3)(1-\alpha)} 2^{m_{0}(1+\alpha p-p)},
$$

for some finite constant $C_{\alpha}$. Hence, setting $\lambda=2^{m_{0}}$, it follows that

$$
\begin{aligned}
& \sum_{m=-\infty}^{\infty} 2^{m} \mid E_{m}\left.\cap Q^{\prime}\right|^{1-\alpha} \\
& \leq 2\left|Q^{\prime}\right|^{1-\alpha} \lambda+C_{\alpha}\|\mathbf{b}\|_{3, p}^{p(1-\alpha)}\left|Q^{\prime}\right|^{(1-p / 3)(1-\alpha)} \lambda^{(1+\alpha p-p)} .
\end{aligned}
$$

The RHS of the last inequality is minimised when $\lambda \sim\|\mathbf{b}\|_{3, p}\left|Q^{\prime}\right|^{-1 / 3}$. We conclude therefore that

$$
\sum_{m=-\infty}^{\infty} 2^{m}\left|E_{m} \cap Q^{\prime}\right|^{1-\alpha} \leq C_{\alpha}\|\mathbf{b}\|_{3, p}\left|Q^{\prime}\right|^{2 / 3-\alpha},
$$

for some finite constant $C_{\alpha}$. Putting this last inequality together with (2.9), (2.10), and (2.11) we conclude that

$$
\begin{aligned}
\int_{Q^{\prime} \cap B_{1 / 4}} & (1 / 4-|x|)^{2}\left[\sum_{n=n_{Q^{\prime}}}^{\infty}|\mathbf{b}(x)|^{1 / 2} S_{n} h(x)\right]^{2} d x \\
\leq & C^{2}\|\mathbf{b}\|_{3, p}^{1 / 2}\left|Q^{\prime}\right|^{1 / 6} h_{Q^{\prime}}\|\mathbf{b}\|_{3, p}^{1 / 2}\left|Q^{\prime}\right|^{\alpha+1 / 6} h_{Q^{\prime}}\|\mathbf{b}\|_{3, p}\left|Q^{\prime}\right|^{2 / 3-\alpha} \\
& =C^{2}\|\mathbf{b}\|_{3, p}^{2}\left|Q^{\prime}\right| h_{Q^{\prime}}^{2}
\end{aligned}
$$

for some constant $C$ depending only on $p$ and $q$. 
Proposition 2.2 follows now from (2.6) and Lemma 2.5 just in the same way as Theorem 1.2 of $[\mathbf{C R}]$ follows from Lemma 2.4 of $[\mathbf{C R}]$. Next we consider the third stage in section 4 of the proof that the operator $Q_{\rho}$ is bounded on $L^{2}$. Let $K_{\rho}$ be an operator on functions $h: B_{1 / 4} \rightarrow \mathbb{C}$ defined by

$$
K_{\rho} h(x)=\int_{B_{1 / 4}} d y G_{D}(x, y)|\mathbf{b}(y)|^{1 / 2} h(y), \quad|x|=\frac{1}{4}(1-\rho),
$$

where $0<\rho<1 / 2$.

Proposition 2.4. For $0<\rho<1 / 2, K_{\rho}$ is a bounded operator from $L_{\text {weight }}^{2}\left(B_{1 / 4}\right)$ to $L^{2}\left(\partial B_{(1-\rho) / 4}\right)$ and the norm of $K_{\rho}$ satisfies an inequality $\left\|K_{\rho}\right\| \leq C\|\mathbf{b}\|_{3, p}^{1 / 2}$, where the constant $C$ depends only on $p>1$.

Proof: We write the operator $K_{\rho}$ as a sum

$$
K_{\rho}=\sum_{n=0}^{\infty} K_{\rho, n}
$$

where

$$
\begin{aligned}
& K_{\rho, 0} h(x)=\int_{B_{1 / 4} \cap\left\{|x-y|<\frac{1}{5} \rho\right\}} d y G_{D}(x, y)|\mathbf{b}(y)|^{1 / 2} h(y), \\
& K_{\rho, n} h(x)=\int_{B_{1 / 4} \cap\left\{\frac{1}{5} \rho 2^{n-1} \leq|x-y|<\frac{1}{5} \rho 2^{n}\right\}} d y G_{D}(x, y)|\mathbf{b}(y)|^{1 / 2} h(y), \\
& n=1,2, \ldots .
\end{aligned}
$$

Evidently

$$
\begin{aligned}
\left|K_{\rho, 0} h(x)\right| & \leq \int_{B_{1 / 4} \cap\left\{|x-y|<\frac{1}{5} \rho\right\}} \frac{|\mathbf{b}(y)|^{1 / 2}|h(y)|}{|x-y|} d y \\
& \leq\left[\int_{\left\{|x-y|<\frac{1}{5} \rho\right\}} \frac{|\mathbf{b}(y)|}{|x-y|} d y\right]^{1 / 2}\left[\int_{B_{1 / 4} \cap\left\{|x-y|<\frac{1}{5} \rho\right\}} \frac{|h(y)|^{2}}{|x-y|} d y\right]^{1 / 2},
\end{aligned}
$$

by the Schwarz inequality. Since $\mathbf{b} \in M_{p}^{3}$ there is a universal constant $C$ such that

$$
\int_{\left\{|x-y|<\frac{1}{5} \rho\right\}} \frac{|\mathbf{b}(y)|}{|x-y|} d y \leq C\|\mathbf{b}\|_{3, p} \rho .
$$


Hence

$$
\begin{aligned}
\int_{|x|=\frac{1}{4}(1-\rho)}\left|K_{\rho, 0} h(x)\right|^{2} d x & \leq C\|\mathbf{b}\|_{3, p} \rho \int_{\left\{|x|=\frac{1}{4}(1-\rho)\right\}} d x \int_{\left\{|x-y|<\frac{1}{5} \rho\right\}} \frac{|h(y)|^{2}}{|x-y|} d y \\
& \leq C_{1}\|\mathbf{b}\|_{3, p} \rho^{2} \int_{\{1 / 4-|y|>\rho / 20\} \cap B_{1 / 4}}|h(y)|^{2} d y \\
& \leq C_{2}\|\mathbf{b}\|_{3, p} \int_{B_{1 / 4}}(1 / 4-|y|)^{2}|h(y)|^{2} d y \\
& =C_{2}\|\mathbf{b}\|_{3, p}\|h\|_{2, \text { weight }}^{2}
\end{aligned}
$$

where $C_{1}$ and $C_{2}$ are universal constants. Hence we have shown that $K_{\rho, 0}$ is a bounded operator and that

$$
\left\|K_{\rho, 0}\right\| \leq C_{2}^{1 / 2}\|\mathbf{b}\|_{3, p}^{1 / 2} .
$$

Next we consider $K_{\rho, n}$ for $n \geq 1$. We use the fact that there is a universal constant $C$ such that if $|x|=(1-\rho) / 4$, then

$G_{D}(x, y) \leq C \rho(1 / 4-|y|) /\left(\rho 2^{n}\right)^{3}, y \in B_{1 / 4} \cap\left\{\frac{1}{5} \rho 2^{n-1} \leq|x-y|<\frac{1}{5} \rho 2^{n}\right\}$.

Applying the Schwarz inequality as we did before we have

$$
\begin{aligned}
& \left|K_{\rho, n} h(x)\right| \\
\leq & \frac{C_{1} \rho}{\left(\rho 2^{n}\right)^{3}}\|\mathbf{b}\|_{3, p}^{1 / 2} \rho 2^{n}\left[\int_{B_{1 / 4} \cap\left\{\frac{1}{5} \rho 2^{n-1} \leq|x-y|<\frac{1}{5} \rho 2^{n}\right\}}(1 / 4-|y|)^{2}|h(y)|^{2} d y\right]^{1 / 2},
\end{aligned}
$$

for some universal constant $C_{1}$. Hence there is a universal constant $C_{2}$ such that

$$
\int_{|x|=\frac{1}{4}(1-\rho)}\left|K_{\rho, n} h(x)\right|^{2} d x \leq C_{2}\|\mathbf{b}\|_{3, p} 2^{-2 n}\|h\|_{2, \text { weight }}^{2} .
$$

It follows that $K_{\rho, n}$ is a bounded operator and

$$
\left\|K_{\rho, n}\right\| \leq C_{2}^{1 / 2}\|\mathbf{b}\|_{3, p}^{1 / 2} 2^{-n}, \quad n \geq 1
$$

Now the boundedness of $K_{\rho}$ follows from the Minkowski inequality

$$
\left\|K_{\rho}\right\| \leq \sum_{n=0}^{\infty}\left\|K_{\rho, n}\right\| \leq C_{2}^{1 / 2}\|\mathbf{b}\|_{3, p}^{1 / 2} \sum_{n=0}^{\infty} 2^{-n}=2 C_{2}^{1 / 2}\|\mathbf{b}\|_{3, p}^{1 / 2}
$$


Proof of Theorem 1.4: Suppose $g$ is in $L^{2}\left(\partial B_{1 / 4}\right)$. Then from Proposition 2.1 and Harnack the function $h(y)=|\mathbf{b}(y)|^{1 / 2} \mathbf{n}(y) \cdot \nabla P g(y)$ is in $L_{\text {weight }}^{2}\left(B_{1 / 4}\right)$ and

$$
\|h\|_{2, \text { weight }} \leq C\|\mathbf{b}\|_{3, p}^{1 / 2}\|g\|_{2},
$$

where $C$ is a constant depending only on $p>1$. By Proposition 2.2 the function $u$ defined by $u=\left(I-T_{\text {sym }}\right)^{-1} h$ is in $L_{\text {weight }}^{2}\left(B_{1 / 4}\right)$ and

$$
\|u\|_{2, \text { weight }} \leq C_{1}\|h\|_{2, \text { weight }},
$$

for some constant $C_{1}$ depending only on $p>1$ provided $\|\mathbf{b}\|_{3, p}$ is sufficiently small. Finally we have $Q_{\rho} g=K_{\rho} u$. Hence Proposition 2.4 and the previous two inequalities tells us that $Q_{\rho}$ is a bounded operator from $L^{2}\left(\partial B_{1 / 4}\right)$ to $L^{2}\left(\partial B_{(1-\rho) / 4}\right)$ provided $\|\mathbf{b}\|_{3, p}$ is sufficiently small and $\left\|Q_{\rho}\right\| \leq C\|\mathbf{b}\|_{3, p}$ for some constant $C$ depending only on $p>1$.

\section{Chapter 3. Fourier Space Localisation}

Our first goal is to prove a version of Theorem 1.5 which takes account of the location of $g$ in Fourier space.

Theorem 3.1. Suppose $f, g$ are in $L^{2}(S)$. Then there exists $\varepsilon>0$ and a constant $C>0$ depending only on $p>1$ such that $\|\mathbf{b}\|_{3, p}<\varepsilon$ implies

$$
\left|\left\langle f, Q_{\rho} E_{k} g\right\rangle\right| \leq C\|\mathbf{b}\|_{3, p}\|f\|_{2}\left\|E_{k} g\right\|_{2} \rho 2^{k}, \quad 0 \leq k<\infty
$$

We can prove Theorem 3.1 by slightly modifying the proof of Theorem 1.4. The main point to observe is that the quantity $2^{k}$ in the estimate (3.1) replaces the weighting factor in the $L^{2}$ norm of Proposition 2.2. We shall therefore need to apply Proposition 2.3 here instead of Proposition 2.2. First we define an operator which is analogous to the operator $A$ of (2.1). Thus for $0<\rho<1 / 2$ let $A_{\rho} f(y)$ be defined on functions $f$ with domain $\partial B_{(1-\rho) / 4}$ by

$$
A_{\rho} f(y)=|\mathbf{b}(y)|^{1 / 2} \frac{4}{\rho} \int_{|x|=(1-\rho) / 4} f(x) G_{D}(x, y) d x, \quad|y|<1 / 4 .
$$

It is evident that

$$
\lim _{\rho \rightarrow 0} A_{\rho} f(y)=A f(y), \quad|y|<1 / 4 .
$$

The following proposition is therefore a generalization of Proposition 2.1. 
Proposition 3.1. For $0<\rho<1 / 2, A_{\rho}$ is a bounded linear operator from $L^{2}\left(\partial B_{(1-\rho) / 4}\right)$ to $L^{2}\left(B_{1 / 4}\right)$. There is a constant $C$ depending only on $p>1$ such that $\left\|A_{\rho}\right\| \leq C\|\mathbf{b}\|_{3, p}^{1 / 2}$.

Proof: Let $\mathcal{U}_{\rho}$ be the spherical shell

$$
\mathcal{U}_{\rho}=\left\{y: \frac{1}{4}(1-3 \rho / 2)<|y|<\frac{1}{4}\right\}
$$

and $\chi_{\rho}$ be the characteristic function of the set $\mathcal{U}_{\rho}$. Let us first consider the operator $K_{\rho}$ defined by

$$
K_{\rho} f(y)=\chi_{\rho}(y) A_{\rho} f(y), \quad y \in B_{1 / 4} .
$$

We show that $K_{\rho}$ is bounded by arguing as in Proposition 2.4. Thus we write

$$
K_{\rho}=\sum_{n=0}^{\infty} K_{\rho, n}
$$

where

$$
\begin{aligned}
& K_{\rho, 0} f(y)=\chi_{\rho}(y)|\mathbf{b}(y)|^{1 / 2} \frac{4}{\rho} \int_{\left\{|x|=\frac{1}{4}(1-\rho),|x-y|<\rho\right\}} f(x) G_{D}(x, y) d x, \\
& K_{\rho, n} f(y)=\chi_{\rho}(y)|\mathbf{b}(y)|^{1 / 2} \frac{4}{\rho} \int_{\left\{|x|=\frac{1}{4}(1-\rho), \rho 2^{n-1} \leq|x-y|<\rho 2^{n}\right\}} f(x) G_{D}(x, y) d x, \\
& n \geq 1 .
\end{aligned}
$$

We have now from the Schwarz inequality that

$$
\left|K_{\rho, 0} f(y)\right|^{2} \leq C \chi_{\rho}(y)|\mathbf{b}(y)| \rho^{-1} \int_{\left\{|x|=\frac{1}{4}(1-\rho),|x-y|<\rho\right\}} \frac{|f(x)|^{2}}{|x-y|} d x,
$$

for some universal constant $C$. Hence

$$
\begin{aligned}
\left\|K_{\rho, 0} f(y)\right\|_{2}^{2} & \leq C \rho^{-1} \int_{|x|=\frac{1}{4}(1-\rho)} d x|f(x)|^{2} \int_{|x-y|<\rho} \frac{|\mathbf{b}(y)|}{|x-y|} d y \\
& \leq C_{1}\|\mathbf{b}\|_{3, p}\|f\|_{2}^{2}
\end{aligned}
$$

for some constant $C_{1}$. We conclude that $\left\|K_{\rho, 0}\right\| \leq C_{1}^{1 / 2}\|\mathbf{b}\|_{3, p}^{1 / 2}$. To estimate $K_{\rho, n}$ for $n \geq 1$ we use the bound

$$
G_{D}(x, y) \leq C \rho /\left(\rho 2^{n}\right)^{2}, \quad|x|=\frac{1}{4}(1-\rho), \quad \rho 2^{n-1} \leq|x-y|<\rho 2^{n},
$$


where $C$ is a universal constant. Hence we have

$$
\begin{aligned}
& \left|K_{\rho, n} f(y)\right|^{2} \\
& \quad \leq C_{1} \chi_{\rho}(y)|\mathbf{b}(y)| \frac{1}{\left(\rho 2^{n}\right)^{2}} \int_{\left\{|x|=\frac{1}{4}(1-\rho), \rho 2^{n-1} \leq|x-y|<\rho 2^{n}\right\}}|f(x)|^{2} d x,
\end{aligned}
$$

for some universal constant $C_{1}$. Hence

$$
\left\|K_{\rho, n} f\right\|_{2}^{2} \leq \frac{C_{1}}{\left(\rho 2^{n}\right)^{2}} \int_{|x|=\frac{1}{4}(1-\rho)} d x|f(x)|^{2} \int_{\mathcal{U}_{\rho} \cap\left\{\rho 2^{n-1} \leq|x-y|<\rho 2^{n}\right\}}|\mathbf{b}(y)| d y .
$$

We have now

$$
\begin{aligned}
& \int_{\mathcal{U}_{\rho} \cap\left\{\rho 2^{n-1} \leq|x-y|<\rho 2^{n}\right\}}|\mathbf{b}(y)| d y \\
& \quad \leq \operatorname{meas}\left[\mathcal{U}_{\rho} \cap\left\{\rho 2^{n-1} \leq|x-y|<\rho 2^{n}\right\}\right]^{1-1 / p}\left[\int_{|x-y|<\rho 2^{n}}|\mathbf{b}(y)|^{p} d y\right]^{1 / p} \\
& \quad \leq C\left(\rho^{3} 2^{2 n}\right)^{1-1 / p}\|\mathbf{b}\|_{3, p}\left(\rho 2^{n}\right)^{3 / p-1} \\
& \quad=C\|\mathbf{b}\|_{3, p} \rho^{2} 2^{n(1+1 / p)}
\end{aligned}
$$

for some universal constant $C$. From the last two inequalities we conclude that there is a universal constant $C_{1}$ such that

$$
\left\|K_{\rho, n}\right\| \leq C_{1}^{1 / 2}\|\mathbf{b}\|_{3, p}^{1 / 2} 2^{-n(1-1 / p) / 2}, \quad n \geq 1
$$

Hence

$$
\left\|K_{\rho}\right\| \leq C_{1}^{1 / 2}\|\mathbf{b}\|_{3, p}^{1 / 2} \sum_{n=0}^{\infty} 2^{-n(1-1 / p) / 2} \leq C_{2}^{1 / 2}\|\mathbf{b}\|_{3, p}^{1 / 2}
$$

for some constant $C_{2}$ depending only on $p>1$.

Next we consider the operator $A_{0}$ defined by

$$
A_{0} f(y)=\left[1-\chi_{\rho}(y)\right] A_{\rho} f(y), \quad y \in B_{1 / 4} .
$$

It is easy to see that there is a universal constant $C$ such that

$$
G_{D}(x, y) \leq C \rho(1 / 4-|y|) /|x-y|^{3}, \quad y \in B_{1 / 4} \backslash \mathcal{U}_{\rho}, \quad|x|=\frac{1}{4}(1-\rho)
$$


Furthermore for $y \in B_{1 / 4} \backslash \mathcal{U}_{\rho}$ one has $1 / 4-|y| \leq C_{1}\left[\frac{1}{4}(1-\rho)-|y|\right]$ for a suitable constant $C_{1}$. Hence the operator $A_{0}$ has a kernel which is bounded by a constant times the kernel of $A$. Applying Proposition 2.1 we conclude that $A_{0}$ is bounded and $\left\|A_{0}\right\| \leq C_{2}^{1 / 2}\|\mathbf{b}\|_{3, p}^{1 / 2}$. Since $A_{\rho}=$ $A_{0}+K_{\rho}$ the result follows from this last inequality and (3.3).

Proposition 3.1 enables us to pull out the factor $\rho$ in the inequality (3.1). Next we address the problem of how to pull out the factor $2^{k}$ in (3.1). The factor occurs due to the effect of the gradient in the expression $\nabla\left(P E_{k} g\right)$. Suppose $y=\left(y_{1}, y_{2}, y_{3}\right) \in \mathbb{R}^{3}$. We shall want to show that

$$
|y| \frac{\partial}{\partial y_{i}} P E_{k} g(y) \simeq 2^{k} P h_{i}(y), \quad|y|<1 / 4, \quad 1 \leq i \leq 3,
$$

where $h_{i}$ is an $L^{2}$ function on $\partial B_{1 / 4}$ with norm comparable to $g$. Furthermore we shall need to show that $h_{i}$ is approximately concentrated in Fourier space on the range of $E_{k}$. To do this we introduce polar coordinates $(r, \theta, \varphi)$ on the ball, $0 \leq r<1 / 4,0<\theta<\pi, 0<\varphi<2 \pi$. We may also assume that $i=3$ in (3.4) and the representation

$$
\frac{\partial}{\partial y_{3}}=\cos \theta \frac{\partial}{\partial r}-\sin \theta \frac{1}{r} \frac{\partial}{\partial \theta} \text {. }
$$

Let $Y_{\ell, m}(\theta, \varphi)$ be the spherical harmonics on the unit sphere. Thus $\ell$ is a nonnegative integer, $m$ is an integer satisfying $-\ell \leq m \leq \ell$ and

$$
\begin{aligned}
-\Delta_{S} Y_{\ell, m} & =\ell(\ell+1) Y_{\ell, m}, \\
\frac{1}{i} \frac{\partial}{\partial \varphi} Y_{\ell, m} & =m Y_{\ell, m} .
\end{aligned}
$$

Now the Poisson kernel applied to the boundary data $Y_{\ell, m}$ yields

$$
P Y_{\ell, m}(r, \theta, \varphi)=(4 r)^{\ell} Y_{\ell, m}(\theta, \varphi) .
$$

Thus

$$
|y| \frac{\partial}{\partial y_{3}} P Y_{\ell, m}(r, \theta, \varphi)=\ell(4 r)^{\ell} \cos \theta Y_{\ell, m}(\theta, \varphi)-(4 r)^{\ell} \sin \theta \frac{\partial}{\partial \theta} Y_{\ell, m}(\theta, \varphi) .
$$

Let $P_{\ell, m}(z), \ell=0,1,2, \ldots, 0 \leq m \leq \ell$ be the associated Legendre functions. Then one has [M, p. 495], for $m \geq 0$,

$$
Y_{\ell, \pm m}(\theta, \varphi)=\sigma_{ \pm m, \ell}\left[\frac{(2 \ell+1)(\ell-m) !}{4 \pi(\ell+m) !}\right]^{1 / 2} P_{\ell, m}(\cos \theta) e^{ \pm i m \varphi}
$$


where $\left|\sigma_{ \pm m, \ell}\right|=1$. If we use the relations

$$
\begin{aligned}
(2 \ell+1) z P_{\ell, m}(z) & =(\ell+m) P_{\ell-1, m}(z)+(\ell+1-m) P_{\ell+1, m}(z), \\
\left(z^{2}-1\right) \frac{d}{d z} P_{\ell, m}(z) & =(\ell-m+1) P_{\ell+1, m}(z)-(\ell+m+1) z P_{\ell, m}(z),
\end{aligned}
$$

to be found in [H, p. 289,290$]$, we may conclude that

$$
\begin{aligned}
\cos \theta Y_{\ell, m}(\theta, \varphi)= & {\left[\frac{(\ell+m)(\ell-m)}{(2 \ell+1)(2 \ell-1)}\right]^{1 / 2} Y_{\ell-1, m}(\theta, \varphi) } \\
& +\left[\frac{(\ell+1+m)(\ell+1-m)}{(2 \ell+3)(2 \ell+1)}\right]^{1 / 2} Y_{\ell+1, m}(\theta, \varphi), \\
\sin \theta \frac{\partial}{\partial \theta} Y_{\ell, m}(\theta, \varphi)= & -(\ell+m+1)\left[\frac{(\ell+m)(\ell-m)}{(2 \ell+1)(2 \ell-1)}\right]^{1 / 2} Y_{\ell-1, m}(\theta, \varphi) \\
& +(\ell-m)\left[\frac{(\ell+1+m)(\ell+1-m)}{(2 \ell+3)(2 \ell-1)}\right]^{1 / 2} Y_{\ell+1, m}(\theta, \varphi) .
\end{aligned}
$$

Hence we have shown that

$$
|y| \frac{\partial}{\partial y_{3}} P Y_{\ell, m}(r, \theta, \varphi)=\ell\left[a r P Y_{\ell-1, m}(r, \theta, \varphi)+b r^{-1} P Y_{\ell+1, m}(r, \theta, \varphi)\right]
$$

where $a, b$ are constants which are bounded by 16 in absolute value. It is easy now to state a rigorous version of (3.4).

Lemma 3.1. Let $g$ be square integrable on the sphere $\partial B_{1 / 4}$, such that $E_{k} g=g$ for some $k \geq 1$. Then for any $i, 1 \leq i \leq 3$, there exist functions $h_{+}, h_{-}$on $\partial B_{1 / 4}$ such that

$$
|y| \frac{\partial}{\partial y_{i}} P g(y)=2^{k}(4|y|) P h_{-}(y)+2^{k}(1 / 4|y|) P h_{+}(y), \quad|y|<1 / 4,
$$

and the functions $h_{+}, h_{-}$satisfy

$$
\begin{array}{ll}
\left(E_{k}+E_{k+1}\right) h_{+}=h_{+}, & \left(E_{k}+E_{k-1}\right) h_{-}=h_{-}, \\
\left\|h_{+}\right\|_{2} \leq C\|g\|_{2}, & \left\|h_{-}\right\|_{2} \leq C\|g\|_{2},
\end{array}
$$

where $C$ is a universal constant. 
Proof of Theorem 3.1: Consider the function $h_{k}$ on $B_{1 / 4}$ defined by

$$
h_{k}(y)=2^{-k}|\mathbf{b}(y)|^{1 / 2} \mathbf{n}(y) \cdot \nabla P E_{k} g(y) .
$$

In view of Lemma 3.1 and Proposition 2.1 the function $h_{k}$ is in $L^{2}\left(B_{1 / 4}\right)$ and

$$
\left\|h_{k}\right\|_{2} \leq C\|\mathbf{b}\|_{3, p}^{1 / 2}\left\|E_{k} g\right\|_{2} .
$$

Next by Proposition 2.3 if $\|\mathbf{b}\|_{3, p}$ is sufficiently small then $u_{k}=(I-$ $\left.T_{\text {sym }}\right)^{-1} h_{k}$ is also in $L^{2}\left(B_{1 / 4}\right)$ and

$$
\left\|u_{k}\right\|_{2} \leq C_{1}\left\|h_{k}\right\|_{2}
$$

for some constant $C_{1} \leq 2$. Observe now that

$$
\left\langle f, Q_{\rho} E_{k} g\right\rangle=\rho 2^{k}\left\langle A_{\rho} f, u_{k}\right\rangle,
$$

where $A_{\rho}$ is the operator (3.2). The result follows now from the last two inequalities and Proposition 3.1.

Next we wish to prove a version of Theorem 1.5 which takes account of both the location of $f$ and $g$ in Fourier space.

Theorem 3.2. Suppose $f, g$ are in $L^{2}(S)$. Then there exists $\varepsilon>0$ and constants $\eta, C>0$ depending only on $p>1$ such that $\|\mathbf{b}\|_{3, p}<\varepsilon$ implies

$$
\left|\left\langle E_{k^{\prime}} f, Q_{\rho} E_{k} g\right\rangle\right| \leq C\|\mathbf{b}\|_{3, p}\left\|E_{k^{\prime}} f\right\|_{2}\left\|E_{k} g\right\|_{2} 2^{\eta\left(k^{\prime}-k\right)}, \quad 0 \leq k, k^{\prime}<\infty .
$$

Evidently Theorem 3.2 is implied by Theorem 1.4 if $k \leq k^{\prime}$ so we shall assume that $k>k^{\prime}$. The basic fact we want to use is that the function $P\left(E_{k} g\right)(y), y \in B_{1 / 4}$ falls off rapidly from the boundary, $|y|=1 / 4$. In fact a simple computation shows that the function is essentially concentrated on the shell $\frac{1}{4}\left(1-2^{-k}\right)<|y|<\frac{1}{4}$. We need to define a norm which is sensitive to this fact. For $h: B_{1 / 4} \rightarrow \mathbb{C}, k=0,1,2, \ldots$ and $\delta>0$ we define a norm $\|h\|_{2, k, \delta}$ by

$$
\|h\|_{2, k, \delta}=\sup _{0 \leq r \leq k}\left[2^{2 r \delta} \int_{\mathcal{U}_{r, k}}|h(y)|^{2} d y\right]^{1 / 2},
$$

where $\mathcal{U}_{r, k}$ are spherical shells given by

$$
\begin{aligned}
& \mathcal{U}_{0, k}=\left\{y: \frac{1}{4}\left(1-2^{-k}\right)<|y|<\frac{1}{4}\right\}, \\
& \mathcal{U}_{r, k}=\left\{y: \frac{1}{4}\left(1-2^{r} 2^{-k}\right)<|y|<\frac{1}{4}\left(1-2^{r-1} 2^{-k}\right)\right\}, \quad 1 \leq r \leq k .
\end{aligned}
$$

Observe that if $k=0$ then one just gets back the $L^{2}$ norm of $h$. 
Lemma 3.2. Suppose $g$ is square integrable on $\partial B_{1 / 4}$ and $A$ is the operator defined by (2.1). Then for $k=0,1,2, \ldots$, and any $\delta>0$ the function $A E_{k} g(y)$ has norm satisfying

$$
\left\|A E_{k} g\right\|_{2, k, \delta} \leq C_{\delta, p}\|\mathbf{b}\|_{3, p}^{1 / 2}\left\|E_{k} g\right\|_{2},
$$

where the constant $C_{\delta, p}$ depends only on $\delta \geq 0, p>1$.

Proof: Evidently

$$
\int_{U_{0, k}}\left|A E_{k} g(y)\right|^{2} d y \leq \int_{B_{1 / 4}}\left|A E_{k} g(y)\right|^{2} d y \leq C_{p}\|g\|_{2}^{2}
$$

by Proposition 2.1. Next for $1 \leq r \leq k$ observe that if $y \in \mathcal{U}_{r, k}$ then

$$
P g(y)=P_{r} g_{r}(y)
$$

where the operator $P_{r}$ acts on functions with domain $\left\{|y|=\frac{1}{4}\left(1-2^{r-1} 2^{-k}\right)\right\}$ and gives the solution of the Dirichlet problem. Thus $u(y)=P_{r} h(y)$ satisfies

$$
\begin{aligned}
\Delta u(y) & =0, \quad|y|<\frac{1}{4}\left(1-2^{r-1} 2^{-k}\right), \\
u(y) & =h(y), \quad|y|=\frac{1}{4}\left(1-2^{r-1} 2^{-k}\right) .
\end{aligned}
$$

It is easy to see that the function $g_{r}$ has $L^{2}$ norm bounded as $\left\|g_{r}\right\|_{2} \leq$ $\exp \left[-c 2^{r}\right]\|g\|_{2}$ for some positive constant $c>0$. Now applying Theorem 1.4 again we have that

$$
\int_{\mathcal{U}_{r, k}}\left|A E_{k} g(y)\right|^{2} d y \leq\left\|g_{r}\right\|_{2}^{2} \leq C_{p} \exp \left[-c 2^{r+1}\right]\|g\|_{2}^{2} .
$$

The result follows now from this last inequality and (3.5).

Next we need to show that the operator $T_{\text {sym }}$ is a bounded operator on the space determined by \|\|$_{2, k, \delta}$. As before we shall define two spaces associated with this norm. First the space $L_{k, \delta}^{2}\left(B_{1 / 4}\right)$ is defined by $h \in L_{k, \delta}^{2}\left(B_{1 / 4}\right)$ if $\|h\|_{2, k, \delta}<\infty$, with norm given by \|\|$_{2, k, \delta}$. The weighted space $L_{k, \delta \text {,weight }}^{2}\left(B_{1 / 4}\right)$ is defined as all $h$ such that the function $f(y)=(1 / 4-|y|) h(y)$ has finite norm $\|f\|_{2, k, \delta}<\infty$. The weighted norm of $h$ is then given by $\|h\|_{2, k, \delta \text {, weight }}=\|f\|_{2, k, \delta}$. We have now two theorems analagous to Propositions 2.2, 2.3. 
Proposition 3.2. There exists $\delta>0$ such that the operator $T_{\mathrm{sym}}$ is bounded on the space $L_{k, \delta \text {, weight }}^{2}\left(B_{1 / 4}\right)$ and there exists a constant $C>0$ depending only on $\delta, p>1$ such that $\left\|T_{\mathrm{sym}}\right\| \leq C\|\mathbf{b}\|_{3, p}$.

Proposition 3.3. There exists $\delta>0$ such that the operator $T_{\mathrm{sym}}$ is bounded on the space $L_{k, \delta}^{2}\left(B_{1 / 4}\right)$ and there exists a constant $C>0$ depending only on $\delta, p>1$ such that $\left\|T_{\mathrm{sym}}\right\| \leq C\|\mathbf{b}\|_{3, p}$.

Proof: We shall first prove Proposition 3.3. Let us suppose $x \in \mathcal{U}_{k, k}$. Then

$$
\begin{aligned}
\left|T_{\text {sym }} h(x)\right| & \leq \frac{|\mathbf{b}(x)|^{1 / 2}}{2 \pi} \sum_{s=0}^{k} \int_{\mathcal{U}_{s, k}} \frac{|\mathbf{b}(y)|^{1 / 2}|h(y)|}{|x-y|^{2}} d y \\
& \leq T_{\text {sym }} h_{1}(x)+C|\mathbf{b}(x)|^{1 / 2} \int_{B_{1 / 4}}|\mathbf{b}(y)|^{1 / 2}|h(y)| d y
\end{aligned}
$$

where $C$ is a universal constant and

$$
\begin{aligned}
h_{1}(y) & =h(y), & & y \in \mathcal{U}_{k-1, k} \cup \mathcal{U}_{k, k}, \\
& =0, & & \text { otherwise. }
\end{aligned}
$$

By the Minkowski inequality we have then

$$
\begin{aligned}
{\left[\int_{\mathcal{U}_{k, k}}\left|T_{\mathrm{sym}} h(x)\right|^{2} d x\right]^{1 / 2} \leq\left\|T_{\mathrm{sym}} h_{1}\right\|_{2} } \\
+C\left[\int_{B_{1 / 4}}|\mathbf{b}(x)| d x\right]^{1 / 2}\left[\int_{B_{1 / 4}}|\mathbf{b}(y)|^{1 / 2}|h(y)| d y\right] .
\end{aligned}
$$

Evidently $\left\|h_{1}\right\|_{2} \leq 2^{-(k-2) \delta}\|h\|_{2, k, \delta}$. On the other hand

$$
\begin{aligned}
\int_{B_{1 / 4}}|\mathbf{b}(y)|^{1 / 2}|h(y)| d y & \leq \sum_{s=0}^{k}\left[\int_{\mathcal{U}_{s, k}}|\mathbf{b}(y)| d y\right]^{1 / 2}\left[\int_{\mathcal{U}_{s, k}}|h(y)|^{2} d y\right]^{1 / 2} \\
& \leq \sum_{s=0}^{k}\left[\int_{\mathcal{U}_{s, k}}|\mathbf{b}(y)| d y\right]^{1 / 2} 2^{-s \delta}\|h\|_{2, k, \delta} \\
& \leq \sum_{s=0}^{k} \operatorname{meas}\left(\mathcal{U}_{s, k}\right)^{(1-1 / p) / 2}\|\mathbf{b}\|_{3, p}^{1 / 2} 2^{-s \delta}\|h\|_{2, k, \delta} \\
& \leq C \sum_{s=0}^{k}\|\mathbf{b}\|_{3, p}^{1 / 2}\|h\|_{2, k, \delta} 2^{(s-k)(1-1 / p) / 2} 2^{-s \delta} \\
& \leq C_{1}\|\mathbf{b}\|_{3, p}^{1 / 2}\|h\|_{2, k, \delta} 2^{-k \delta}
\end{aligned}
$$


provided $\delta<(1-1 / p) / 2$. Now from Proposition 2.3 and the last four inequalities we conclude that

$$
\left[\int_{\mathcal{U}_{k, k}}\left|T_{\mathrm{sym}} h(x)\right|^{2} d x\right]^{1 / 2} \leq C\|\mathbf{b}\|_{3, p}\|h\|_{2, k, \delta} 2^{-k \delta}
$$

where the constant $C$ depends only on $p>1$ and $\delta>0$.

We can easily deal with the case of the integral of $T_{\text {sym }} h$ over $\mathcal{U}_{0, k}$ by observing that

$$
\left[\int_{\mathcal{U}_{0, k}}\left|T_{\text {sym }} h(x)\right|^{2} d x\right]^{1 / 2} \leq\left\|T_{\text {sym }} h\right\|_{2} \leq C\|\mathbf{b}\|_{3, p}\|h\|_{2} \leq C_{\delta}\|\mathbf{b}\|_{3, p}\|h\|_{2, k, \delta}
$$

by Proposition 2.3. Thus we are left to deal with integrals over $\mathcal{U}_{r, k}$ with $1 \leq r \leq k-1$. Define the function $h_{1}(y)$ by

$$
\begin{aligned}
h_{1}(y) & =h(y), & y \in \mathcal{U}_{s, k}, \quad s \geq r-1 \\
& =0, \quad & \text { otherwise. }
\end{aligned}
$$

Next for $s \leq r-2$ and integer $n$ satisfying $2 \leq n \leq k-r+4$ let $g_{s, n}(x)$ be the function

$$
g_{s, n}(x)=\frac{|\mathbf{b}(x)|^{1 / 2}}{2 \pi} 2^{2 n} \int_{\mathcal{U}_{s, k} \cap\left\{2^{-n}<|x-y|<2^{-n+1}\right\}}|\mathbf{b}(y)|^{1 / 2}|h(y)| d y .
$$

Then we have the inequality

$$
\left|T_{\mathrm{sym}} h(x)\right| \leq\left|T_{\mathrm{sym}} h_{1}(x)\right|+\sum_{s=0}^{r-2} \sum_{n=2}^{k-r+4} g_{s, n}(x), \quad x \in \mathcal{U}_{r, k}
$$

It follows by the Minkowski inequality that

$$
\begin{aligned}
{\left[\int_{\mathcal{U}_{r, k}}\left|T_{\mathrm{sym}} h(x)\right|^{2} d x\right]^{1 / 2} \leq } & {\left[\int_{\mathcal{U}_{r, k}}\left|T_{\mathrm{sym}} h_{1}(x)\right|^{2} d x\right]^{1 / 2} } \\
& +\sum_{s=0}^{r-2} \sum_{n=2}^{k-r+4}\left[\int_{\mathcal{U}_{r}, k} g_{s, n}(x)^{2} d x\right]^{1 / 2}
\end{aligned}
$$


By Proposition 2.3 we have

$$
\begin{aligned}
{\left[\int_{\mathcal{U}_{r, k}}\left|T_{\mathrm{sym}} h_{1}(x)\right|^{2} d x\right]^{1 / 2} } & \leq\left\|T_{\mathrm{sym}} h_{1}\right\|_{2} \\
& \leq C\|\mathbf{b}\|_{3, p}\left\|h_{1}\right\|_{2} \\
& \leq C_{1}\|\mathbf{b}\|_{3, p}\|h\|_{2, k, \delta} 2^{-r \delta} .
\end{aligned}
$$

Observe next that for any $z$,

$$
\begin{aligned}
& \int_{\mathcal{U}_{r, k} \cap\left\{|x-z|<2^{-n}\right\}} g_{s, n}(x)^{2} d x \leq \frac{2^{4 n}}{(2 \pi)^{2}} \int_{\mathcal{U}_{r, k} \cap\left\{|x-z|<2^{-n}\right\}}|\mathbf{b}(x)| d x \\
& {\left[\int_{\mathcal{U}_{s, k} \cap\left\{|z-y|<2^{-n+2}\right\}}|\mathbf{b}(y)|^{1 / 2}|h(y)| d y\right]^{2} . }
\end{aligned}
$$

From Holder's inequality we have

$$
\begin{aligned}
& \int_{\mathcal{U}_{r, k} \cap\left\{|x-z|<2^{-n}\right\}}|\mathbf{b}(x)| d x \\
& \leq \operatorname{meas}\left[\mathcal{U}_{r, k} \cap\left\{|x-z|<2^{-n}\right\}\right]^{1-1 / p}\left[\int_{|x-z|<2^{-n}}|\mathbf{b}(x)|^{p} d x\right]^{1 / p} \\
& \leq C\|\mathbf{b}\|_{3, p} 2^{(r-k-2 n)(1-1 / p)} 2^{-n(3 / p-1)} .
\end{aligned}
$$

Similarly we have

$$
\begin{gathered}
{\left[\int_{\mathcal{U}_{s, k} \cap\left\{|z-y|<2^{-n+2}\right\}}|\mathbf{b}(y)|^{1 / 2}|h(y)| d y\right]^{2} \leq\left[\int_{\mathcal{U}_{s, k} \cap\left\{|z-y|<2^{-n+2}\right\}}|\mathbf{b}(y)| d y\right]} \\
{\left[\int_{\mathcal{U}_{s, k} \cap\left\{|z-y|<2^{-n+2}\right\}}|h(y)|^{2} d y\right] \leq C} \\
{\left[\int_{\mathcal{U}_{s, k} \cap\left\{|z-y|<\|_{3, p} 2^{(s-k-2 n)(1-1 / p)} 2^{-n(3 / p-1)}\right.}|h(y)|^{2} d y .\right.}
\end{gathered}
$$

The last three inequalities imply then that

$$
\begin{aligned}
\int_{\mathcal{U}_{r, k}} g_{s, n}(x)^{2} d x \leq & C\|\mathbf{b}\|_{3, p}^{2} 2^{4 n} 2^{(r-k-2 n)(1-1 / p)} 2^{-n(3 / p-1)} \\
& 2^{(s-k-2 n)(1-1 / p)} 2^{-n(3 / p-1)} \int_{\mathcal{U}_{s, k}}|h(y)|^{2} d y \\
\leq & C\|\mathbf{b}\|_{3, p}^{2} 2^{-2 r \delta}\|h\|_{2, k, \delta}^{2} 2^{-2(k-r-n)(1-1 / p)} 2^{(s-r)(1-1 / p-2 \delta)} .
\end{aligned}
$$


It follows then from the previous inequality and (3.6), (3.7) that if $\delta<(1-1 / p) / 2$ then

$$
\left[\int_{\mathcal{U}_{r, k}}\left|T_{\mathrm{sym}} h(x)\right|^{2} d x\right]^{1 / 2} \leq C\|\mathbf{b}\|_{3, p}\|h\|_{2, k, \delta} 2^{-r \delta}
$$

for some constant $C$ depending only on $\delta$ and $p>1$. This completes the proof of Proposition 3.3. Proposition 3.2 follows in an exactly analogous way from Proposition 2.2.

Next let us consider the operator $K_{\rho}$ defined by (2.12). Let $f$ be a function in $L^{2}\left(\partial B_{(1-\rho) / 4}\right)$. We define the function $M_{\rho} f(y), y \in B_{1 / 4}$ by

$$
\int_{|x|=\frac{1}{4}(1-\rho)} f(x) K_{\rho} h(x) d x=\int_{B_{1 / 4}}\left(\frac{1}{4}-|y|\right) h(y) M_{\rho} f(y) d y,
$$

for $h \in L_{\text {weight }}^{2}\left(B_{1 / 4}\right)$. Explicitly we have

$$
M_{\rho} f(y)=\int_{|x|=\frac{1}{4}(1-\rho)} d x f(x) G_{D}(x, y)|\mathbf{b}(y)|^{1 / 2}\left(\frac{1}{4}-|y|\right)^{-1} .
$$

In view of Proposition 2.4 we see that $M_{\rho}$ is a bounded operator from $L^{2}\left(\partial B_{(1-\rho) / 4)}\right)$ to $L^{2}\left(B_{1 / 4}\right)$. Furthermore there is a constant $C$ depending only on $p>1$ such that

$$
\left\|M_{\rho}\right\| \leq C\|\mathbf{b}\|_{3, p}^{1 / 2}
$$

provided $0<\rho<1 / 2$. We shall need to more accurately estimate the effect of $M_{\rho}$ on a function $f$ which is concentrated in a band of Fourier space. In particular we have the following:

Proposition 3.4. Suppose $k, k^{\prime}$ are nonnegative integers and $0<\rho<1 / 2$. Then there exists $\delta>0$ and a constant $C$ depending only on $p>1$ such that for $f \in L^{2}\left(\partial B_{(1-\rho) / 4}\right)$,

$$
\int_{\mathcal{U}_{0, k}}\left|M_{\rho} E_{k^{\prime}} f(y)\right|^{2} d y \leq C\|\mathbf{b}\|_{3, p}\left\|E_{k^{\prime}} f\right\|_{2}^{2} 2^{-2\left(k-k^{\prime}\right) \delta} .
$$

Proof: In view of the inequality (3.8) we may assume that $k>k^{\prime}$. We shall first show that we may also assume $2^{-k^{\prime}}>\rho$. This will follow from the inequality

$$
\int_{\mathcal{U}_{0, k}}\left|M_{\rho} f(y)\right|^{2} d y \leq C\|\mathbf{b}\|_{3, p}\|f\|_{2}^{2}\left(2^{-k} / \rho\right)^{2 \delta} .
$$


Observe that it is sufficient to prove (3.9) under the condition $2^{-k}<\rho / 2$. In that case we write

$$
M_{\rho} f(y)=\sum_{n=0}^{\infty} g_{n}(y), \quad y \in \mathcal{U}_{0, k}
$$

where

$$
\begin{aligned}
& g_{0}(y)=\int_{\left\{|x|=\frac{1}{4}(1-\rho),|x-y|<\rho\right\}} d x f(x) G_{D}(x, y)|\mathbf{b}(y)|^{1 / 2}(1 / 4-|y|)^{-1}, \\
& g_{n}(y)=\int_{\left\{|x|=\frac{1}{4}(1-\rho), 2^{n-1} \rho<|x-y|<2^{n} \rho\right\}} d x f(x) G_{D}(x, y)|\mathbf{b}(y)|^{1 / 2}(1 / 4-|y|)^{-1} .
\end{aligned}
$$

Since $2^{-k}<\rho / 2$ we have

$$
\begin{array}{r}
\left|g_{n}(y)\right| \leq \frac{C \rho}{\left(\rho 2^{n}\right)^{3}}|\mathbf{b}(y)|^{1 / 2} \int_{\left\{|x|=\frac{1}{4}(1-\rho),|x-y|<2^{n} \rho\right\}}|f(x)| d x \\
\leq \frac{C_{1} \rho}{\left(\rho 2^{n}\right)^{2}}|\mathbf{b}(y)|^{1 / 2}\left[\int_{\left\{|x|=\frac{1}{4}(1-\rho),|x-y|<2^{n} \rho\right\}}|f(x)|^{2} d x\right]^{1 / 2}, \\
n=0,1,2, \ldots,
\end{array}
$$

for some universal constants $C, C_{1}$ by the Schwarz inequality. This last inequality implies

$$
\begin{aligned}
& \int_{\mathcal{U}_{0, k}}\left|g_{n}(y)\right|^{2} d y \\
& \quad \leq \frac{C_{1}^{2} \rho^{2}}{\left(\rho 2^{n}\right)^{4}} \int_{\left\{|x|=\frac{1}{4}(1-\rho)\right\}} d x|f(x)|^{2} \int_{\mathcal{U}_{0, k} \cap\left\{|x-y|<2^{n} \rho\right\}}|\mathbf{b}(y)| d y .
\end{aligned}
$$

Now if we estimate

$$
\begin{aligned}
& \int_{\mathcal{U}_{0, k}} \cap\left\{|x-y|<2^{n} \rho\right\} \\
& \quad \leq \operatorname{meas}\left[\mathcal{U}_{0, k} \cap\left\{|x-y|<2^{n} \rho\right\}\right]^{1-1 / p}\|\mathbf{b}\|_{3, p}\left(2^{n} \rho\right)^{3 / p-1} \\
& \quad \leq C\|\mathbf{b}\|_{3, p}\left(2^{n} \rho\right)^{1+1 / p} 2^{-k(1-1 / p)}
\end{aligned}
$$


we can conclude that

$$
\begin{aligned}
& {\left[\int_{\mathcal{U}_{0, k}}\left|g_{n}(y)\right|^{2} d y\right]^{1 / 2}} \\
& \quad \leq C_{1}\|\mathbf{b}\|_{3, p}^{1 / 2}\|f\|_{2}\left(2^{-k} / \rho\right)^{(1-1 / p) / 2} 2^{-n(3-1 / p) / 2}
\end{aligned}
$$

for some universal constant $C_{1}$. Hence from (3.10) and the Minkowski inequality it follows that (3.9) holds with $\delta=(1-1 / p) / 2$.

We can assume now that $2^{-k^{\prime}}>\rho, k>k^{\prime}$. Let $f_{k^{\prime}}(x)$ be the function

$$
f_{k^{\prime}}=2^{-4 k^{\prime}}\left(-\Delta_{S}+2^{2 k^{\prime}}\right)^{2} E_{k^{\prime}} f
$$

where $\Delta_{S}$ is the Laplacian on the unit sphere. Evidently there is a universal constant $C$ such that $\left\|f_{k^{\prime}}\right\|_{2} \leq C\left\|E_{k^{\prime}} f\right\|_{2}$. Furthermore $E_{k^{\prime}} f$ can be written in terms of $f_{k^{\prime}}$ by

$$
E_{k^{\prime}} f(x)=\int_{\left|x^{\prime}\right|=\frac{1}{4}(1-\rho)} H_{k^{\prime}}\left(x, x^{\prime}\right) f_{k^{\prime}}\left(x^{\prime}\right) d x^{\prime},
$$

where $H_{k^{\prime}}\left(x, x^{\prime}\right)$ is the kernel of the operator $2^{4 k^{\prime}}\left(-\Delta_{S}+2^{2 k^{\prime}}\right)^{-2}$. It is well known $[\mathbf{C H}]$ that there are constants $C, c>0$ such that

$$
0 \leq H_{k^{\prime}}\left(x, x^{\prime}\right) \leq C 2^{2 k^{\prime}} \exp \left[-c\left|x-x^{\prime}\right| / 2^{-k^{\prime}}\right]
$$

Again we write

$$
M_{\rho} E_{k^{\prime}} f(y)=\sum_{n=0}^{\infty} g_{n}(y), \quad y \in \mathcal{U}_{0, k}
$$

where

$$
\begin{aligned}
g_{0}(y)= & \int_{\left\{|x|=\frac{1}{4}(1-\rho),\left|x^{\prime}\right|=\frac{1}{4}(1-\rho),\left|x^{\prime}-y\right|<2^{-k^{\prime}}\right\}} \\
& d x d x^{\prime} H_{k^{\prime}}\left(x, x^{\prime}\right) f_{k^{\prime}}\left(x^{\prime}\right) G_{D}(x, y)|\mathbf{b}(y)|^{1 / 2}(1 / 4-|y|)^{-1}, \\
g_{n}(y)= & \int_{\left\{|x|=\frac{1}{4}(1-\rho),\left|x^{\prime}\right|=\frac{1}{4}(1-\rho), 2^{n-1-k^{\prime}}<\left|x^{\prime}-y\right|<2^{n-k^{\prime}}\right\}} \\
& d x d x^{\prime} H_{k^{\prime}}\left(x, x^{\prime}\right) f_{k^{\prime}}\left(x^{\prime}\right) G_{D}(x, y)|\mathbf{b}(y)|^{1 / 2}(1 / 4-|y|)^{-1},
\end{aligned}
$$


if $n \geq 1$. Observe now that

$$
\begin{aligned}
\int_{|x|=\frac{1}{4}(1-\rho)} & d x H_{k^{\prime}}\left(x, x^{\prime}\right) G_{D}(x, y)(1 / 4-|y|)^{-1} \\
& \leq C 2^{2 k^{\prime}} \int_{|x|=\frac{1}{4}(1-\rho)} d x G_{D}(x, y)(1 / 4-|y|)^{-1} \leq C_{1} 2^{2 k^{\prime}}
\end{aligned}
$$

for some universal constant $C_{1}$. Hence

$$
\left|g_{0}(y)\right| \leq C_{1} 2^{2 k^{\prime}} \int_{\left\{\left|x^{\prime}\right|=\frac{1}{4}(1-\rho),\left|x^{\prime}-y\right|<2^{-k^{\prime}}\right\}} d x^{\prime}\left|f_{k^{\prime}}\left(x^{\prime}\right)\right||\mathbf{b}(y)|^{1 / 2}
$$

Now if $2^{n-1-k^{\prime}}<\left|x^{\prime}-y\right|<2^{n-k^{\prime}}, n \geq 1$, then one easily sees that

$$
\int_{|x|=\frac{1}{4}(1-\rho)} d x H_{k^{\prime}}\left(x, x^{\prime}\right) G_{D}(x, y)(1 / 4-|y|)^{-1} \leq C 2^{2 k^{\prime}-3 n}
$$

for some universal constant $C_{2}$. Hence if $n \geq 1$ we have the inequality

$$
\begin{aligned}
& \left|g_{n}(y)\right| \\
& \leq C_{2} 2^{2 k^{\prime}-3 n} \int_{\left\{\left|x^{\prime}\right|=\frac{1}{4}(1-\rho),\left|x^{\prime}-y\right|<2^{n-k^{\prime}}\right\}} d x^{\prime}\left|f_{k^{\prime}}\left(x^{\prime}\right)\right||\mathbf{b}(y)|^{1 / 2} .
\end{aligned}
$$

We can now bound the integrals of $\left|g_{n}(y)\right|^{2}$ over $\mathcal{U}_{0, k}$ exactly as in (3.12) by using the inequalities (3.13), (3.14). There is therefore a universal constant $C_{1}$ such that

$$
\begin{aligned}
& {\left[\int_{\mathcal{U}_{0, k}}\left|g_{n}(y)\right|^{2} d y\right]^{1 / 2}} \\
& \quad \leq C_{1}\|\mathbf{b}\|_{3, p}^{1 / 2}\left\|f_{k^{\prime}}\right\|_{2} 2^{\left(k^{\prime}-k\right)(1-1 / p) / 2} 2^{-n(3-1 / p) / 2}, \quad n \geq 0 .
\end{aligned}
$$

Since $\left\|f_{k^{\prime}}\right\|_{2} \leq C\left\|E_{k^{\prime}} f\right\|_{2}$ the result follows as before. 
Proof of Theorem 3.2: Let $h(y)=|\mathbf{b}(y)|^{1 / 2} \mathbf{n}(y) \cdot \nabla P E_{k} g(y), y \in B_{1 / 4}$. Then from the Harnack principle and Lemma 3.2 it is easy to see that $h$ is in the space $L_{k, \delta \text {, weight }}^{2}\left(B_{1 / 4}\right)$ for every $\delta>0$ and

$$
\|h\|_{2, k, \delta, \text { weight }} \leq C_{\delta, p}\|\mathbf{b}\|_{3, p}^{1 / 2}\left\|E_{k} g\right\|_{2}
$$

where the constant $C_{\delta, p}$ depends only on $\delta, p>1$. Now by Proposition 3.2 one sees that if $\|\mathbf{b}\|_{3, p}$ is sufficiently small then the function $\xi(y)=(1 / 4-|y|)\left(I-T_{\mathrm{sym}}\right)^{-1} h(y)$ is in $L_{k, \delta}^{2}\left(B_{1 / 4}\right)$ and

$$
\|\xi\|_{2, k, \delta} \leq C_{\delta, p}\|\mathbf{b}\|_{3, p}^{1 / 2}\left\|E_{k} g\right\|_{2}
$$

for some suitable constant $C_{\delta, p}$. Observe next that

$$
\begin{aligned}
\left|\left\langle E_{k^{\prime}} f, Q_{\rho} E_{k} g\right\rangle\right| & =\left|\int_{B_{1 / 4}} \xi(y) M_{\rho} E_{k^{\prime}} f(y) d y\right| \\
& \leq \sum_{r=0}^{k}\left[\int_{\mathcal{U}_{r, k}}|\xi(y)|^{2} d y\right]^{1 / 2}\left[\int_{\mathcal{U}_{r, k}}\left|M_{\rho} E_{k^{\prime}} f(y)\right|^{2} d y\right]^{1 / 2} .
\end{aligned}
$$

If we use now Proposition 3.4 we have that

$$
\begin{aligned}
\left|\left\langle E_{k^{\prime}} f, Q_{\rho} E_{k} g\right\rangle\right| \leq & \sum_{r=0}^{k} 2^{-r \delta}\|\xi\|_{2, k, \delta}\left[\int_{\mathcal{U}_{r, k}}\left|M_{\rho} E_{k^{\prime}} f(y)\right|^{2} d y\right]^{1 / 2} \\
\leq & \sum_{r=0}^{k-k^{\prime}} 2^{-r \delta}\|\xi\|_{2, k, \delta} C^{1 / 2}\|\mathbf{b}\|_{3, p}^{1 / 2}\left\|E_{k^{\prime}} f\right\|_{2} 2^{-\left(k-k^{\prime}-r\right) \delta^{\prime}} \\
& +\sum_{r=k-k^{\prime}+1}^{k} 2^{-r \delta}\|\xi\|_{2, k, \delta} C^{1 / 2}\|\mathbf{b}\|_{3, p}^{1 / 2}\left\|E_{k^{\prime}} f\right\|_{2} \\
\leq & C_{1}\|\mathbf{b}\|_{3, p}^{1 / 2}\|\xi\|_{2, k, \delta}\left\|E_{k^{\prime}} f\right\|_{2} 2^{-\left(k-k^{\prime}\right) \delta} \\
\leq & C_{2}\|\mathbf{b}\|_{3, p}\left\|E_{k} g\right\|_{2}\left\|E_{k^{\prime}} f\right\|_{2} 2^{-\left(k-k^{\prime}\right) \delta}
\end{aligned}
$$

for constants $C_{1}, C_{2}$ depending only on $p>1$ provided we choose $0<\delta<\delta^{\prime}$ appropriately. 
The proof of Theorem 1.5 will be complete if we can prove:

Theorem 3.3. Suppose $f, g$ are in $L^{2}(S)$. Then there exists $\varepsilon>0$ and constants $\eta, C>0$ depending only on $p>1$ such that if $\|\mathbf{b}\|_{3, p}<\varepsilon$ then

$\left|\left\langle E_{k^{\prime}} f, Q_{\rho} E_{k} g\right\rangle\right| \leq C\|\mathbf{b}\|_{3, p}\left\|E_{k^{\prime}} f\right\|_{2}\left\|E_{k} g\right\|_{2} \rho 2^{k} 2^{\eta\left(k-k^{\prime}\right)}, \quad 0 \leq k, k^{\prime}<\infty$.

The main work to be done to prove this last proposition is to show that Proposition 3.4 also holds for the operator $A_{\rho}$ defined by (3.2). Thus we have the following:

Proposition 3.5. Suppose $k, k^{\prime}$ are nonnegative integers and $0<\rho<1 / 2$. Then there exists $\delta>0$ and a constant $C$ depending only on $p>1$ such that for $f \in L^{2}\left(\partial B_{(1-\rho) / 4}\right)$,

$$
\int_{\mathcal{U}_{0, k}}\left|A_{\rho} E_{k^{\prime}} f(y)\right|^{2} d y \leq C\|\mathbf{b}\|_{3, p}\left\|E_{k^{\prime}} f\right\|_{2}^{2} 2^{-2\left(k-k^{\prime}\right) \delta} .
$$

Proof: We proceed in the same way as in Proposition 3.4. By Proposition 3.1 we can assume that $k \geq k^{\prime}$. Next we show that one may also assume $2^{-k^{\prime}}>\rho$. This follows from the inequality

$$
\int_{\mathcal{U}_{0, k}}\left|A_{\rho} f(y)\right|^{2} d y \leq C\|\mathbf{b}\|_{3, p}\|f\|_{2}^{2}\left(2^{-k} / \rho\right)^{2 \delta} .
$$

Observe that it is sufficient to prove (3.15) under the condition $2^{-k}<$ $\rho / 2$. In that case we write

$$
A_{\rho} f(y)=\sum_{n=0}^{\infty} g_{n}(y), \quad y \in \mathcal{U}_{0, k}
$$

where

$$
\begin{aligned}
& g_{0}(y)=\frac{4}{\rho} \int_{\left\{|x|=\frac{1}{4}(1-\rho),|x-y|<\rho\right\}} d x f(x) G_{D}(x, y)|\mathbf{b}(y)|^{1 / 2}, \\
& g_{n}(y)=\frac{4}{\rho} \int_{\left\{|x|=\frac{1}{4}(1-\rho), 2^{n-1} \rho<|x-y|<2^{n} \rho\right\}} d x f(x) G_{D}(x, y)|\mathbf{b}(y)|^{1 / 2}, \quad n \geq 1 .
\end{aligned}
$$

Since $2^{-k}<\rho / 2$ we have

$$
\left|g_{n}(y)\right| \leq \frac{C \rho}{\left(\rho 2^{n}\right)^{3}}|\mathbf{b}(y)|^{1 / 2} \int_{\left\{|x|=\frac{1}{4}(1-\rho),|x-y|<2^{n} \rho\right\}}|f(x)| d x .
$$


Since this last inequality is exactly the same as (3.11) we can conclude that the theorem holds in the case of $2^{-k^{\prime}} \leq \rho$. To deal with the case of $2^{-k^{\prime}}>\rho$ we proceed again as in Proposition 3.4. The functions $g_{n}$ are now defined by

$$
\begin{array}{r}
g_{0}(y)=\frac{4}{\rho} \int_{\left\{|x|=\frac{1}{4}(1-\rho),\left|x^{\prime}\right|=\frac{1}{4}(1-\rho),\left|x^{\prime}-y\right|<2^{-k^{\prime}}\right\}} \\
d x d x^{\prime} H_{k^{\prime}}\left(x, x^{\prime}\right) f_{k^{\prime}}\left(x^{\prime}\right) G_{D}(x, y)|\mathbf{b}(y)|^{1 / 2}, \\
g_{n}(y)=\frac{4}{\rho} \int_{\left\{|x|=\frac{1}{4}(1-\rho),\left|x^{\prime}\right|=\frac{1}{4}(1-\rho), 2^{n-1-k^{\prime}}<\left|x^{\prime}-y\right|<2^{n-k^{\prime}}\right\}} \begin{array}{r}
d x d x^{\prime} H_{k^{\prime}}\left(x, x^{\prime}\right) f_{k^{\prime}}\left(x^{\prime}\right) G_{D}(x, y)|\mathbf{b}(y)|^{1 / 2},
\end{array}
\end{array}
$$

if $n \geq 1$. Evidently one has

$$
\begin{aligned}
& \frac{4}{\rho} \int_{|x|=\frac{1}{4}(1-\rho)} d x H_{k^{\prime}}\left(x, x^{\prime}\right) G_{D}(x, y) \\
& \quad \leq \frac{4 C 2^{2 k^{\prime}}}{\rho} \int_{|x|=\frac{1}{4}(1-\rho)} d x G_{D}(x, y) \leq C_{1} 2^{2 k^{\prime}}
\end{aligned}
$$

for some universal constant $C_{1}$. Similarly

$$
\frac{4}{\rho} \int_{|x|=\frac{1}{4}(1-\rho)} d x H_{k^{\prime}}\left(x, x^{\prime}\right) G_{D}(x, y) \leq C_{2} 2^{2 k^{\prime}-3 n}
$$

for some universal constant $C_{2}$ if $2^{n-1-k^{\prime}}<\left|x^{\prime}-y\right|<2^{n-k^{\prime}}, n \geq 1$. Now, using these last two estimates the proof of the theorem is identical to the proof of Proposition 3.4.

Proof of Theorem 3.3: Let $h(y)=|\mathbf{b}(y)|^{1 / 2} \mathbf{n}(y) \cdot \nabla P E_{k} g(y), y \in B_{1 / 4}$. From Lemmas 3.1, 3.2 it follows that $h$ is in the space $L_{k, \delta}^{2}\left(B_{1 / 4}\right)$ for every $\delta>0$ and

$$
\|h\|_{2, k, \delta} \leq C_{\delta, p}\|\mathbf{b}\|_{3, p}^{1 / 2} 2^{k}\left\|E_{k} g\right\|_{2}
$$

where the constant $C_{\delta, p}$ depends only on $\delta, p>1$. Now by Proposition 3.3 one sees that if $\|\mathbf{b}\|_{3, p}$ is sufficiently small then the function $\xi(y)=\left(I-T_{\mathrm{sym}}\right)^{-1} h(y)$ is in $L_{k, \delta}^{2}\left(B_{1 / 4}\right)$ and

$$
\|\xi\|_{2, k, \delta} \leq C_{\delta, p}\|\mathbf{b}\|_{3, p}^{1 / 2} 2^{k}\left\|E_{k} g\right\|_{2}
$$


for some suitable constant $C_{\delta, p}$. Observe next that

$$
\left|\left\langle E_{k^{\prime}} f, Q_{\rho} E_{k} g\right\rangle\right|=\left|\rho \int_{B_{1 / 4}} \xi(y) A_{\rho} E_{k^{\prime}} f(y) d y\right| .
$$

The rest of the proof follows now from (3.16) and Proposition 3.5 in exactly the same way as Theorem 3.2 follows from Proposition 3.4.

\section{Chapter 4. Proof of Theorem 1.3}

We first define the density $f_{\rho, \mathbf{b}}$ in terms of the density $f$. To do this we consider the Dirichlet problem

$$
\begin{aligned}
(\Delta+\mathbf{b}(y) \cdot \nabla) v(y) & =0, \quad y \in B_{1 / 4}, \\
v(y) & =g(y), \quad y \in \partial B_{1 / 4} .
\end{aligned}
$$

Formally $v(y)$ is given by the formula

$$
v(y)=P g(y)+Q g(y), \quad y \in B_{1 / 4},
$$

where $P$ is the Poisson integral (1.4) and $Q g$ is defined by (1.5). Now $v$ can be represented in terms of the diffusion process $X_{\mathbf{b}}(t)$ by the expression

$$
v(y)=E_{y}\left[g\left(X_{\mathbf{b}}(\tau)\right)\right], \quad y \in B_{1 / 4},
$$

where $\tau$ is the first hitting time on $\partial B_{1 / 4}$ for the process started at $X_{\mathbf{b}}(0)=y$. It is clear then that if we regard the density $f$ as a function on $\partial B_{(1-\rho) / 4}$ and the density $f_{\rho, \mathbf{b}}$ as a function on $\partial B_{1 / 4}$, then

$$
\begin{aligned}
\int_{\partial B_{(1-\rho) / 4}} f(y) v(y) d y / \text { normalisation } & \\
& =\int_{\partial B_{1 / 4}} f_{\rho, \mathbf{b}}(y) g(y) d y / \text { normalisation, }
\end{aligned}
$$

where the normalisations are chosen so that the measures are probability measures. It follows therefore, on going back to regarding $f$ and $f_{\rho, \mathbf{b}}$ as functions on the unit sphere $S$ that

$$
\left\langle f_{\rho, \mathbf{b}}, g\right\rangle=\left\langle f, P_{\rho} g+Q_{\rho} g\right\rangle, \quad g \in L^{2}(S),
$$

where $P_{\rho} g(y)=P g(y), y \in \partial B_{(1-\rho) / 4}$. Hence $f_{\rho, \mathbf{b}}=P_{\rho}^{*} f+Q_{\rho}^{*} f$, where $P_{\rho}^{*}$ and $Q_{\rho}^{*}$ are the formal adjoints of $P_{\rho}, Q_{\rho}$ respectively. 
We can analyse the operator $P_{\rho}$ precisely since we know its eigenfunctions. In fact if $Y_{l, m}(\theta, \phi), 0 \leq \theta<\pi, 0 \leq \phi<2 \pi$, is a spherical harmonic and we take $g=Y_{l, m}$ then $P g(y)=(4|y|)^{l} Y_{l, m}(\theta, \phi), y \in B_{1 / 4}$. Hence

$$
P_{\rho} Y_{l, m}=(1-\rho)^{l} Y_{l, m}
$$

It follows in particular that $P_{\rho}$ is selfadjoint, whence $P_{\rho}=P_{\rho}^{*}$. We also have that $P_{\rho} 1=P_{\rho} Y_{0,0}=1$. Hence for any $f \in L^{2}(S)$ we have that

$$
\left\|P_{\rho} f-A v f\right\|_{2} \leq(1-\rho)\|f-A v f\|_{2} .
$$

Theorem 1.2 follows from (4.2) and the fact that $\left\|Q_{\rho}\right\| \leq C\|\mathbf{b}\|_{3, p}$ where $C$ depends only on $p, \rho$. In fact

$$
\begin{aligned}
\left\|f_{\rho, \mathbf{b}}-A v f_{\rho, \mathbf{b}}\right\|_{2} & =\left\|P_{\rho} f+Q_{\rho}^{*} f-A v f\right\|_{2} \\
& \leq\left\|P_{\rho} f-A v f\right\|_{2}+\left\|Q_{\rho}^{*} f\right\|_{2} \\
& \leq(1-\rho)\|f-A v f\|_{2}+C\|\mathbf{b}\|_{3, p}\|f\|_{2} .
\end{aligned}
$$

Suppose now $\|f-A v f\|_{2} \leq \delta|A v f|$. Then $\|f\|_{2} \leq(1+\delta)|A v f|$. Hence the last inequality yields

$$
\begin{aligned}
\left\|f_{\rho, \mathbf{b}}-A v f_{\rho, \mathbf{b}}\right\|_{2} & \leq(1-\rho) \delta|A v f|+C\|\mathbf{b}\|_{3, p}(1+\delta)|A v f| \\
& =\left[1-\rho+C\|\mathbf{b}\|_{3, p}\left(1+\delta^{-1}\right)\right] \delta\left|A v f_{\rho, \mathbf{b}}\right|
\end{aligned}
$$

since $A v f=A v f_{\rho, \mathbf{b}}$. Theorem 1.2 follows from the last inequality by choosing $\|\mathbf{b}\|_{3, p}$ sufficiently small.

Theorem 1.3 follows by a similar argument from Theorem 1.5. Since $Y_{l, m}$ is an eigenfunction of $-\Delta_{S}$ with eigenvalue $l(l+1), l=0,1,2, \ldots$, it follows from (4.1) that there is a universal constant $c>0$ with

$$
\left\|E_{k} P_{\rho} f\right\|_{2} \leq\left\{1-c \min \left[\rho 2^{k}, 1\right]\right\}\left\|E_{k} f\right\|_{2}, \quad k \geq 1 .
$$

The inequality (4.3) plays the same role in the proof of Theorem 1.3 as (4.2) plays in the proof of Theorem 1.2. By the Minkowski inequality we have

$$
\begin{aligned}
\left\|E_{k} f_{\rho, \mathbf{b}}\right\|_{2} & =\left\|E_{k} P_{\rho} f+E_{k} Q_{\rho}^{*} f\right\|_{2} \\
& \leq\left\|E_{k} P_{\rho} f\right\|_{2}+\left\|E_{k} Q_{\rho}^{*} f\right\|_{2} .
\end{aligned}
$$


Theorem 1.5 yields an appropriate estimate on $\left\|E_{k} Q_{\rho}^{*} f\right\|_{2}$, which when combined with (4.3) proves Theorem 1.3. To see this let us assume $\|f-A v f\|_{2, \nu} \leq \delta|A v f|$. Then

$$
\left\|E_{k} f\right\|_{2} \leq \delta|A v f| / 2^{\nu k}, \quad k=1,2, \ldots .
$$

Now, for some $g$ satisfying $\left\|E_{k} g\right\|_{2}=1$,

$$
\left\|E_{k} Q_{\rho}^{*} f\right\|_{2}=\left\langle f, Q_{\rho} E_{k} g\right\rangle \leq \sum_{k^{\prime}=0}^{\infty}\left|\left\langle E_{k^{\prime}} f, Q_{\rho} E_{k} g\right\rangle\right| .
$$

Hence from Theorem 1.5 and (4.4) we have,

$$
\begin{aligned}
\left\|E_{k} Q_{\rho}^{*} f\right\|_{2} \leq & C\|\mathbf{b}\|_{3, p}|A v f| \min \left[\rho 2^{k}, 1\right] 2^{-\eta k} \\
& +\sum_{k^{\prime}=1}^{k} C\|\mathbf{b}\|_{3, p} \delta|A v f| 2^{-\nu k^{\prime}} \min \left[\rho 2^{k}, 1\right] 2^{\eta\left(k-k^{\prime}\right)} \\
& +\sum_{k^{\prime}=k+1}^{\infty} C\|\mathbf{b}\|_{3, p} \delta|A v f| 2^{-\nu k^{\prime}} \min \left[\rho 2^{k}, 1\right] .
\end{aligned}
$$

Note that the first term on the right in the last inequality comes from the $k^{\prime}=0$ term on the right in (4.5). Hence if $\eta>\nu$ we have that

$$
\begin{aligned}
\left\|E_{k} Q_{\rho}^{*} f\right\|_{2} & \leq C\|\mathbf{b}\|_{3, p}|A v f| \min \left[\rho 2^{k}, 1\right] \\
& \left\{2^{-\eta k}+\delta 2^{(\eta-\nu)(k+1)} 2^{-\eta k} /\left[2^{(\eta-\nu)}-1\right]+\delta 2^{-\nu(k+1)} /\left[1-2^{-\nu}\right]\right\} .
\end{aligned}
$$

We conclude that

$$
\left\|E_{k} Q_{\rho}^{*} f\right\|_{2} \leq C(\delta)\|\mathbf{b}\|_{3, p} \delta|A v f| \min \left[\rho 2^{k}, 1\right] 2^{-\nu k}, \quad k \geq 1,
$$

where the constant $C(\delta)$ depends only on $\delta$. Combining this last inequality with (4.3) we have that

$$
\left\|E_{k} f_{\rho, \mathbf{b}}\right\|_{2} \leq\left\{1+\left[C(\delta)\|\mathbf{b}\|_{3, p}-c\right] \min \left[\rho 2^{k}, 1\right]\right\} \delta|A v f| 2^{-\nu k}, \quad k \geq 1 .
$$

The theorem follows now by choosing $\|\mathbf{b}\|_{3, p}$ sufficiently small so that $C(\delta)\|\mathbf{b}\|_{3, p}<c$.

Acknowledgement. The authors would like to thank Paul Federbush and Mark Pinsky for helpful conversations on spherical harmonics. 


\title{
References
}

[CR] J. CONLON AND J. Redondo, Estimates on the solution of an elliptic equation related to Brownian motion with drift, Rev. Mat. Iberoamericana 11 (1995), 1-65.

[CO] J. CONlON AND P. Olsen, Estimates on the solution of an elliptic equation related to Brownian motion with drift II, Rev. Mat. Iberoamericana 13 (1997), 567-711.

[O] P. Olsen, Fractional Integration, Morrey Spaces and a Schrodinger Equation, Comm. Partial Differential Equations 20 (1995), 2005-2055.

[M] A. Messiah, "Quantum Mechanics," Volume I, John Wiley, 1958.

[H] E. Hobson, "The Theory of Spherical and Ellipsoidal Harmonics," Chelsea, New York, 1955.

[A] D. Adams, A note on Riesz potentials, Duke Math. J. 42 (1975), 765-778.

[CF] F. Chiarenza And M. Frasca, Morrey spaces and HardyLittlewood maximal function, Rend. Mat. Appl. (7) 7 (1988), 273-279.

[CH] I. Chavel, "Eigenvalues in Riemannian Geometry" Academic Press, 1984.

[SV] D. STROOCK AND S. VARADHAN, "Multidimensional diffusion processes," Springer, 1979.

\author{
Department of Mathematics \\ University of Michigan \\ Ann Arbor, MI 48109-1003 \\ U.S.A.
}

Rebut el 26 de gener de 1998 